\title{
Stationary variational estimates for the effective response and field fluctuations in nonlinear composites
}

\author{
Pedro Ponte Castañeda* \\ Department of Mechanical Engineering and Applied Mechanics, University of Pennsylvania, \\ Philadelphia, PA 19104-6315, USA
}

\begin{abstract}
This paper presents a variational method for estimating the effective constitutive response of composite materials with nonlinear constitutive behavior. The method is based on a stationary variational principle for the macroscopic potential in terms of the corresponding potential of a linear comparison composite (LCC) whose properties are the trial fields in the variational principle. When used in combination with estimates for the LCC that are exact to second order in the heterogeneity contrast, the resulting estimates for the nonlinear composite are also guaranteed to be exact to second-order in the contrast. In addition, the new method allows full optimization with respect to the properties of the LCC, leading to estimates that are fully stationary and exhibit no duality gaps. As a result, the effective response and field statistics of the nonlinear composite can be estimated directly from the appropriately optimized linear comparison composite. By way of illustration, the method is applied to a porous, isotropic, power-law material, and the results are found to compare favorably with earlier bounds and estimates. However, the basic ideas of the method are expected to work for broad classes of composites materials, whose effective response can be given appropriate variational representations, including more general elasto-plastic and soft hyperelastic composites and polycrystals.

Keywords: Composites, Polycrystals, Viscoplasticity, Nonlinear Homogenization, Variational approximations
\end{abstract}

\footnotetext{
${ }^{*}$ Corresponding author

Email address: ponte@seas. upenn.edu (Pedro Ponte Castañeda)
} 


\section{Introduction}

Homogenization methods for nonlinear composite materials have come a long way since the early works of Taylor (1938) and Hill (1965) to estimate the effective behavior of plastically deforming polycrystals. Thus, building on a nonlinear generalization by Willis (1983) of the variational principles of Hashin and Shtrikman (1963) for linear-elastic composites, rigorous bounds of the Hashin-Shtrikman type were obtained by Talbot and Willis (1985) in conductivity and Willis (1991) in viscoplasticity. Later, new variational principles based on the use of a 'linear comparison composite' (LCC) were proposed by Ponte Castañeda (1991, 1992), and used to generate improved bounds and more general estimates for nonlinear composites. The idea of the method was to rewrite the local energy as the sum of the energy of the LCC plus a certain 'error' function consisting of the smallest difference between the linear and nonlinear energies. An alternative method has been developed by Suquet (1992, 1993) by means of Hölder's inequality for special classes of pure power-law composites. In addition, Suquet (1995) has shown that the 'variational' method of Ponte Castañeda (1991) could be given an alternative interpretation as a 'modified secant' approach, thus establishing a link with the second moments of the local fields in the LCC.

In an attempt to improve on these 'variational' bounds, and in particular, to generate estimates that are exact to second order in the heterogeneity contrast, and therefore agree with the perturbation expansions of Suquet and Ponte Castañeda (1993) for weakly heterogeneous nonlinear composites, Ponte Castañeda (1996) proposed a 'second-order' variational method, which made use of a more general LCCs incorporating eigenstresses or polarizations, in addition to the elasticity moduli of the phases of the LCC. More specifically, the eigenstresses were selected optimally in the context of the variational method, but the elastic moduli were set - in ad hoc fashion - equal to the tangent moduli of the nonlinear phases (evaluated at the phase averages of the fields in the LCC). Earlier attempts making use of such more general LCCs were advanced by Talbot and Willis (1992), essentially by combining the Talbot-Willis and Ponte Castañeda procedures, but they were designed to produce boundsusually the same as the earlier variational bounds - due to the use of the extremal points of the 'error' function characterizing the differences between the actual nonlinear composite and the fictitious LCC used in the variational procedures, and were not exact to second- 
order in the contrast. In fact, as further clarified by Ponte Castañeda and Willis (1999), the improvements associated with the 'tangent second-order' estimates of Ponte Castañeda (1996) were made possible by the use of additional 'stationary' (e.g., saddle) points in the above-mentioned error function. Thus, while the 'second-order' estimates are not bounds, they provide improved estimates at least to second-order in the heterogeneity contrast.

Nevertheless, it was later found by Leroy and Ponte Castañeda (2001) that the 'tangent second-order' estimates could violate the rigorous bounds provided by the earlier 'variational' method when the contrast and field fluctuations are large, as is the case for porous materials near the percolation limit. Motivated by this finding, Ponte Castañeda (2002a) proposed an improved 'second-order' method that makes use of a 'generalized secant' linearization of the nonlinear constitutive relations, incorporating dependence on both the first and second moments of the relevant fields in the LCC. The method arises from a generalization of the stationary variational approach of Ponte Castañeda (1996), but insists in the optimality of the moduli of the LCC, as in the 'variational linear comparison' method. However, the method could not be made stationary with respect to the eigenstrains of the LCC, which created some issues concerning the choice of these parameters (e.g., Idiart and Ponte Castañeda, 2006; Rekik et al., 2015). In any event, it was recognized in this and other works (Idiart and Ponte Castañeda, 2003; Idiart et al., 2006; Idiart and Ponte Castañeda, 2007a) that the second-order method could also be used to extract estimates for the phase averages and covariance of the field fluctuations in the phases of the nonlinear composite. Such information is useful for obtaining improved descriptions of microstructure evolution in composite materials and polycrystals that are subjected to finite-deformation processes, as well as for developing statistical theories of damage nucleation and evolution in heterogeneous material systems.

In spite of the improved accuracy of the 'second-order' method of Ponte Castañeda (2002a) relative to the earlier 'variational' method of Ponte Castañeda (1991), the method has certain deficiencies, which, as already mentioned, arise from the lack of full optimality of the properties of the LCC, and complicate its use in applications. These include the facts that the macroscopic constitutive relation and fields statistics cannot be obtained directly from the LCC, and that the estimates resulting from the primary and complementary vari- 
ational statements are different (i.e., there is a 'duality gap'). In turn, these observations strongly suggest possible further improvements in the accuracy of its predictions. In this work, we propose a new 'second-order' variational method, where the properties of the LCC can be fully optimized. This leads to 'fully stationary' estimates that are still exact to second order in the contrast, but have all the advantages of the earlier 'variational' estimates in that the macroscopic constitutive relation and fields statistics of the nonlinear composite can be conveniently expressed in terms of the corresponding quantities for the suitably optimized LCC. Finally, we show explicitly by means of a simple application for a porous, isotropic, power-law, material that the new 'fully stationary' second-order estimates do not exhibit a duality gap and provide more robust estimates than the earlier estimates of Ponte Castañeda (2002b) for the same problem.

Throughout this paper, scalars are denoted by italic Roman, $a$, or Greek letters, $\alpha$; vectors by boldface Roman letters, b; second-order tensors by boldface italic Roman letters, $\boldsymbol{C}$, or boldface Greek letters, $\boldsymbol{\alpha}$; and fourth-order tensors by bared letters, $\mathbb{P}$. Where necessary, conventional index notation is adopted, e.g., $b_{i}, C_{i j}$ and $P_{i j k l}$ are the Cartesian components of the vector $\mathbf{b}$, second order-tensor $\boldsymbol{C}$ and fourth-order tensor $\mathbb{P}$, respectively. In addition, the symbols $\cdot$ and $\otimes$ are used to represent the inner and dyadic (tensor) products, such that, for example, $\mathbf{a} \cdot \mathbf{b}=a_{i} b_{i}, \boldsymbol{C} \cdot \boldsymbol{D}=C_{i j} D_{i j}$, and $\mathbf{a} \otimes \mathbf{b}=a_{i} b_{j}, \boldsymbol{C} \otimes \boldsymbol{D}=C_{i j} D_{k l}$.

\section{Macroscopic response and field statistics}

The focus of this work will be on nonlinear heterogeneous materials composed of $N$ homogeneous phases $\Omega^{(r)}(r=1, \ldots, N)$, which are distributed randomly in a specimen occupying a volume $\Omega$ at a length scale that is much smaller than the size of the specimen and the scale of variation of the loading conditions. The phase materials could be different, as the metal and ceramic constituents in a metal-matrix composite, or could be the same, except for the orientation, as in the grains in a metal polycrystal. Their constitutive behavior will be characterized by energy functions, or potentials, $w^{(r)}(r=1, \ldots, N)$, assumed in this work to be (strictly) convex and (piecewise) smooth, such that the local stress-strain relation 
for the heterogeneous material is given by

$$
\boldsymbol{\sigma}=\frac{\partial w}{\partial \boldsymbol{\epsilon}}(\mathbf{x}, \boldsymbol{\epsilon}), \quad w(\mathbf{x}, \boldsymbol{\epsilon})=\sum_{r=1}^{N} \chi^{(r)}(\mathbf{x}) w^{(r)}(\boldsymbol{\epsilon}),
$$

where the functions $\chi^{(r)}$ are equal to 1 if the position vector $\mathbf{x}$ is inside phase $r$ (i.e., $\mathbf{x} \in \Omega^{(r)}$ ) and zero otherwise. Alternatively, the constitutive behavior of the heterogeneous material could be characterized by the complementary energy functions, or strain potentials, $u^{(r)}$ $(r=1, \ldots, N)$, such that

$$
\boldsymbol{\epsilon}=\frac{\partial u}{\partial \boldsymbol{\sigma}}, \quad u(\mathbf{x}, \boldsymbol{\sigma})=\sum_{r=1}^{N} \chi^{(r)}(\mathbf{x}) u^{(r)}(\boldsymbol{\sigma})
$$

It is convenient to introduce the symbols $\langle$.$\rangle and \langle.\rangle^{(r)}$ to denote volume averages over the composite $(\Omega)$ and over phase $r\left(\Omega^{(r)}\right)$, respectively, so that

$$
\langle w(\mathbf{x}, \boldsymbol{\epsilon})\rangle=\sum_{r=1}^{N} c^{(r)}\left\langle w^{(r)}(\boldsymbol{\epsilon})\right\rangle^{(r)}, \quad\langle u(\mathbf{x}, \boldsymbol{\sigma})\rangle=\sum_{r=1}^{N} c^{(r)}\left\langle u^{(r)}(\boldsymbol{\sigma})\right\rangle^{(r)}
$$

where the scalars $c^{(r)}=\left\langle\chi^{(r)}\right\rangle$ denote the volume fractions of the phases, and where it is recognized that the strain $\boldsymbol{\epsilon}$ and stress fields $\boldsymbol{\sigma}$ may be non-uniform within the phases of the composite.

The complementary potentials $u^{(r)}$ are related to the potentials $w^{(r)}$ via the Legendre transformation:

$$
u^{(r)}(\boldsymbol{\sigma})=\operatorname{stat}_{\boldsymbol{\epsilon}}\left\{\boldsymbol{\sigma} \cdot \boldsymbol{\epsilon}-w^{(r)}(\boldsymbol{\epsilon})\right\}, \quad \text { so that } u(\mathbf{x}, \boldsymbol{\sigma})=\underset{\boldsymbol{\epsilon}}{\operatorname{stat}}\{\boldsymbol{\sigma} \cdot \boldsymbol{\epsilon}-w(\mathbf{x}, \boldsymbol{\epsilon})\}
$$

where the stat(ionarity) operations mean setting the derivative of the terms inside the curly brackets with respect to $\boldsymbol{\epsilon}$ equal to zero, solving for $\boldsymbol{\epsilon}$ as a function of $\boldsymbol{\sigma}$, and substituting the result back inside the brackets to obtain a function of $\boldsymbol{\sigma}$. In view of the assumed convexity (and smoothness) of the potential $w$, the stationarity condition uniquely determines $\boldsymbol{\epsilon}$ as a function of $\boldsymbol{\sigma}$, and the complementary potential $u$ can also be shown to be convex (van Tiel, 1984). However, it should be noted, for later reference, that the above definition still works even when the function $w$ is not convex (see Sewell, 1987). In such a case, there are multiple stationary points in the definition (4) of the Legendre transform $w^{*}$ of $w$, implying that the complementary potential $u$ becomes multiple valued. However, this transformation still has 
the remarkable property that the Legendre transform of the Legendre transform recovers the original function (i.e., $w^{* *}=w$ ), provided that care is exercised when dealing with the various branches of the multi-valued function $w^{*}$.

The relations (1) and (5) can be used to describe several nonlinear constitutive models, including nonlinear infinitesimal elasticity (or deformation theory of plasticity), in which case $\boldsymbol{\epsilon}$ and $\boldsymbol{\sigma}$ are identified with the infinitesimal strain and stress, respectively, as well as viscoplasticity, in which case the associated deformations are finite and $\boldsymbol{\epsilon}$ and $\boldsymbol{\sigma}$ are associated with the Eulerian strain rate and Cauchy stress, respectively. For simplicity, in this work, the results will be presented in the context of elasticity, and $\boldsymbol{\epsilon}$ and $\boldsymbol{\sigma}$ will simply be referred to as the strain and stress. However, it should be kept in mind that all the results can be translated to viscoplasticity with the above-mentioned re-interpretation of $\boldsymbol{\epsilon}$ and $\boldsymbol{\sigma}$.

The behavior of the constituent phases is often assumed to be isotropic and incompressible, such that the phase potentials take the form

$$
u^{(r)}(\boldsymbol{\sigma})=\phi^{(r)}\left(\sigma_{e}\right)
$$

where $\sigma_{e}=\sqrt{\frac{3}{2} s \cdot s}$ denotes the equivalent von Mises stress and $\mathbf{s}$ the stress deviator. Alternatively, the behavior of the constituents could be assumed to anisotropic and incompressible, as in single-crystal viscoplasticity, such that

$$
u^{(r)}(\boldsymbol{\sigma})=\sum_{k=1}^{K^{(r)}} \phi_{(k)}^{(r)}\left(\tau_{(k)}^{(r)}\right),
$$

where the convex functions $\phi_{(k)}^{(r)}\left(k=1, \cdots, K^{(r)}\right)$ characterize the response of the $K^{(r)}$ slip systems in a crystal with orientation $\mathbf{Q}^{(r)}$ and depend on the resolved shear (or Schmid) stresses

$$
\tau_{(k)}^{(r)}=\boldsymbol{\sigma} \cdot \boldsymbol{\mu}_{(k)}^{(r)}, \quad \text { where } \quad \boldsymbol{\mu}_{(k)}^{(r)}=\frac{1}{2}\left(\boldsymbol{n}_{(k)}^{(r)} \otimes \boldsymbol{m}_{(k)}^{(r)}+\boldsymbol{m}_{(k)}^{(r)} \otimes \boldsymbol{n}_{(k)}^{(r)}\right) .
$$

Here the $\boldsymbol{\mu}_{(k)}^{(r)}$ are second-order tensors with $\boldsymbol{n}_{(k)}^{(r)}$ and $\boldsymbol{m}_{(k)}^{(r)}$ denoting the unit vectors normal to the slip plane and along the slip direction of the $k$ th system, respectively, for a crystal with orientation $\mathbf{Q}^{(r)}$.

As is well-known (e.g., Hutchinson, 1976; Willis, 1989; Ponte Castañeda and Suquet, 1998), the macroscopic constitutive behavior of the composite is determined by the macro- 
scopic or homogenized potential

$$
\widetilde{W}(\overline{\boldsymbol{\epsilon}})=\min _{\boldsymbol{\epsilon} \in \mathcal{K}}\langle w(\mathbf{x}, \boldsymbol{\epsilon})\rangle=\operatorname{stat}_{\boldsymbol{\epsilon} \in \mathcal{K}}\langle w(\mathbf{x}, \boldsymbol{\epsilon})\rangle
$$

where $\mathcal{K}$ the set of kinematically admissible strain (rate) tensors $\boldsymbol{\epsilon}$, such that there is a displacement (velocity) field $\mathbf{v}$ satisfying $\boldsymbol{\epsilon}=\frac{1}{2}\left(\nabla \mathbf{v}+\nabla \mathbf{v}^{T}\right)$ in $\Omega$ and $\mathbf{v}=\overline{\boldsymbol{\epsilon}} \mathbf{x}$ on $\partial \Omega$. The second equality in expression (8) follows from the fact that the strain potentials $w^{(r)}$ have been assumed to be (strictly) convex (and to satisfy appropriate growth conditions), so that the unique minimum is attained at the stationary point determined by the condition that the first variational derivative is equal to zero. This condition corresponds to the Euler-Lagrange equation for the variational problem, as given by the equilibrium equation $\nabla \cdot \boldsymbol{\sigma}=\mathbf{0}$, with the stress $\boldsymbol{\sigma}$ determined in terms of the strain $\boldsymbol{\epsilon}$ via expression (1). Thus, $\widetilde{W}$ physically corresponds to the energy stored in the composite when subjected to an affine displacement (velocity) on the boundary with prescribed average strain (rate) $\overline{\boldsymbol{\epsilon}}=\langle\boldsymbol{\epsilon}\rangle$. It is then known (e.g., Ponte Castañeda and Suquet, 1998) that the average stress $\overline{\boldsymbol{\sigma}}=\langle\boldsymbol{\sigma}\rangle$ is related to the average strain (rate) $\overline{\boldsymbol{\epsilon}}$ via the relation

$$
\overline{\boldsymbol{\sigma}}=\frac{\partial \widetilde{W}}{\partial \overline{\boldsymbol{\epsilon}}}
$$

A dual formulation making use of the local complementary potential $u$, leads to the effective constitutive relation for the nonlinear composite

$$
\overline{\boldsymbol{\epsilon}}=\frac{\partial \widetilde{U}}{\partial \overline{\boldsymbol{\sigma}}}
$$

where $\widetilde{U}$ is the macroscopic complementary potential for the composite, defined by

$$
\widetilde{U}(\overline{\boldsymbol{\sigma}})=\min _{\boldsymbol{\sigma} \in \mathcal{S}}\langle u(\mathbf{x}, \boldsymbol{\sigma})\rangle=\operatorname{stat}_{\boldsymbol{\sigma} \in \mathcal{S}}\langle u(\mathbf{x}, \boldsymbol{\sigma})\rangle .
$$

In this relation,

$$
\mathcal{S}=\{\boldsymbol{\sigma}, \operatorname{div} \boldsymbol{\sigma}=\mathbf{0} \text { in } \Omega,\langle\boldsymbol{\sigma}\rangle=\overline{\boldsymbol{\sigma}}\}
$$

denotes the set of statically admissible stresses. Again, under the above-mentioned convexity hypotheses on the $w^{(r)}$, the two formulations are exactly equivalent in the sense of Legendre duality: $\widetilde{U}=\widetilde{W}^{*}$. 
In general, the effective potentials $\widetilde{W}$ and $\widetilde{U}$ are difficult to compute, because they involve sets of nonlinear partial differential equations with randomly oscillating coefficients. In this work, new approximations will be developed for these potentials by making use of variational principles for suitably defined 'linear comparison composites.' The resulting approximations will be required to satisfy the bounds of Ponte Castañeda (1991) and to recover exactly to second-order in the contrast the perturbation expansions of Suquet and Ponte Castañeda (1993).

It is useful at this point to recall some definitions of the field statistics in the composite for later use. Thus, the averages of the stress and strain-rate over phase $r$ are defined by $\overline{\boldsymbol{\sigma}}^{(r)}=\langle\boldsymbol{\sigma}\rangle^{(r)}$ and $\overline{\boldsymbol{\epsilon}}^{(r)}=\langle\boldsymbol{\epsilon}\rangle^{(r)}$, and are such that $\overline{\boldsymbol{\sigma}}=\sum_{r=1}^{N} c^{(r)} \overline{\boldsymbol{\sigma}}^{(r)}$ and $\overline{\boldsymbol{\epsilon}}=\sum_{r=1}^{N} c^{(r)} \overline{\boldsymbol{\epsilon}}^{(r)}$. The equivalent measures associated with $\overline{\boldsymbol{\sigma}}^{(r)}$ and $\overline{\boldsymbol{\epsilon}}^{(r)}$ are defined by: $\bar{\sigma}_{e}^{(r)}=\sqrt{\frac{3}{2} \overline{\mathbf{s}}^{(r)} \cdot \overline{\mathbf{s}}^{(r)}}$, where $\overline{\mathbf{s}}^{(r)}$ is the average stress deviator in phase $r$, and $\bar{\varepsilon}_{e}^{(r)}=\sqrt{\frac{2}{3} \overline{\boldsymbol{\epsilon}}^{(r)} \cdot \overline{\boldsymbol{\epsilon}}^{(r)}}$, respectively. In addition, the second moments of the stress and strain rate over phase $r$ are given by $\langle\boldsymbol{\sigma} \otimes \boldsymbol{\sigma}\rangle^{(r)}$ and $\langle\boldsymbol{\epsilon} \otimes \boldsymbol{\epsilon}\rangle^{(r)}$, while the corresponding phase fluctuation covariance tensors are given by

$$
\mathbb{C}_{\boldsymbol{\sigma}}^{(r)} \doteq\left\langle\left(\boldsymbol{\sigma}-\overline{\boldsymbol{\sigma}}^{(r)}\right) \otimes\left(\boldsymbol{\sigma}-\overline{\boldsymbol{\sigma}}^{(r)}\right)\right\rangle^{(r)}=\langle\boldsymbol{\sigma} \otimes \boldsymbol{\sigma}\rangle^{(r)}-\overline{\boldsymbol{\sigma}}^{(r)} \otimes \overline{\boldsymbol{\sigma}}^{(r)}
$$

and similarly for $\mathbb{C}_{\epsilon}^{(r)}$. In particular, use will be made here of the standard deviation of the von Mises stress and the equivalent plastic strain-rate over phase $r$ :

$$
\mathrm{SD}^{(r)}\left(\sigma_{e}\right)=\sqrt{\left\langle\sigma_{e}^{2}-\left(\bar{\sigma}_{e}^{(r)}\right)^{2}\right\rangle^{(r)}}, \quad \mathrm{SD}^{(r)}\left(\varepsilon_{e}\right)=\sqrt{\left\langle\varepsilon_{e}^{2}-\left(\bar{\varepsilon}_{e}^{(r)}\right)^{2}\right\rangle^{(r)}}
$$

Analytical expressions for the derivation of the first and second moments of the stress and strain fields in the heterogeneous composite have been given by Idiart and Ponte Castañeda (2007a). The idea is to perturb the potential of the given phase by means of suitable terms that are linear, or quadratic in the appropriate field, and to make use of the above variational formulation to generate corresponding estimates for the homogenized potentials of the perturbed problem, which can then be differentiated with respect to the coefficients of the perturbing terms. Idiart and Ponte Castañeda (2007a) have also shown that when the 'variational' (secant) linear comparison method of Ponte Castañeda (1991) is used to estimate the effective potential of the nonlinear heterogeneous material in terms of the potential of a certain linear comparison composite (LCC), the first and second moments of the fields 
over the phases can be estimated directly from the corresponding moments in the LCC. On the other hand, Idiart and Ponte Castañeda (2007a) have shown that when the 'generalized secant' linear comparison method of Ponte Castañeda (2002a) is used to estimate the effective potential of the nonlinear heterogeneous material, the resulting estimates for the first and second moments of the fields over the phases of the nonlinear heterogeneous material do not coincide with the corresponding first and second moments in the LCC; certain additional correction terms are needed in account of the lack of full stationary in the 'generalized secant' linear comparison method of Ponte Castañeda (2002a). One of the primary objectives of this work is to develop fully stationary second-order estimates of the generalized secant type, such that the first and second moments of the fields over the phases of the nonlinear heterogeneous material can also be estimated directly from the corresponding moments in the LCC-without the need for correction terms.

\section{Fully stationary second-order variational estimates}

\subsection{Linear comparison composite}

To set the stage for the variational estimates to be developed in this work for nonlinear composites, we introduce a linear comparison composite (LCC) with the same microstructure, as defined by the functions $\chi^{(r)}$, for the actual nonlinear composite, but with quadratic strain potentials for the $N$ phases, as defined by

$$
u_{L}^{(r)}(\boldsymbol{\sigma})=\frac{1}{2} \boldsymbol{\sigma} \cdot \mathbb{M}^{(r)} \boldsymbol{\sigma}+\boldsymbol{\gamma}^{(r)} \cdot \boldsymbol{\sigma}
$$

where the $\mathbb{M}^{(r)}$ are uniform compliance tensors, and the $\gamma^{(r)}$ are uniform eigenstrains (or 'thermal' strains) of the phases. Differentiation with respect to $\boldsymbol{\sigma}$ shows that the stress-strain relation of this material is indeed linear, i.e., $\boldsymbol{\epsilon}=\mathbb{M}^{(r)} \boldsymbol{\sigma}+\boldsymbol{\gamma}^{(r)}$. Defining the strain potential for the linear comparison composite via

$$
u_{L}(\mathbf{x}, \boldsymbol{\sigma})=\sum_{r=1}^{N} \chi^{(r)}(\mathbf{x}) u_{L}^{(r)}(\boldsymbol{\sigma})
$$

we can then write the effective, or homogenized strain potential for the composite as

$$
\widetilde{U}_{L}(\overline{\boldsymbol{\sigma}})=\underset{\boldsymbol{\sigma} \in \mathcal{S}}{\operatorname{stat}}\left\langle u_{L}(\mathbf{x}, \boldsymbol{\sigma})\right\rangle=\underset{\boldsymbol{\sigma} \in \mathcal{S}}{\operatorname{stat}} \sum_{r=1}^{N} c^{(r)}\left\langle u_{L}^{(r)}(\boldsymbol{\sigma})\right\rangle^{(r)} .
$$


It is well-known(e.g., Laws, 1973; Willis, 1981) that the effective potential $\widetilde{U}_{L}$ may be written in the form

$$
\widetilde{U}_{L}(\overline{\boldsymbol{\sigma}})=\frac{1}{2} \overline{\boldsymbol{\sigma}} \cdot \widetilde{\mathbb{M}} \overline{\boldsymbol{\sigma}}+\tilde{\boldsymbol{\gamma}} \cdot \overline{\boldsymbol{\sigma}}+\frac{1}{2} \tilde{g}
$$

where $\widetilde{\mathbb{M}}, \tilde{\gamma}$ and $\tilde{g}$ are the effective compliance, effective eigenstrain and effective potential at zero stress, as discussed in more detail in Appendix A. Of course, the values of these effective variables will depend not only on the properties of the phases, but also on the specific microstructure. In this work, it will be assumed that estimates, such as the Willis estimate (Willis, 1977; Ponte Castañeda and Willis, 1995), or the self-consistent estimates (Hershey, 1954; Kröner, 1958) are available for this problem, depending on the specific microstructure, or class of microstructures, of interest. The idea here is to make use of such linear estimates to generate corresponding estimates for composites with nonlinear properties, as defined in the previous section, exhibiting identical microstructures (or classes of microstructures).

It is also well-known (see, e.g., Bobeth and Diener, 1987; Parton and Buryachenko, 1990; Ponte Castañeda and Suquet, 1998) that the first and second moments of the stress in the LCC can be obtained from $\widetilde{U}_{L}$ via the identities

$$
\langle\boldsymbol{\sigma}\rangle^{(r)}=\frac{1}{c^{(r)}} \frac{\partial \widetilde{U}_{L}}{\partial \boldsymbol{\gamma}^{(r)}} \quad \text { and } \quad\langle\boldsymbol{\sigma} \otimes \boldsymbol{\sigma}\rangle^{(r)}=\frac{2}{c^{(r)}} \frac{\partial \widetilde{U}_{L}}{\partial \mathbb{M}^{(r)}}
$$

\subsection{Stationary variational estimates}

Returning to the nonlinear composite with strain potentials $u^{(r)}$ for the $N$ phases, we make use of the potentials $u_{L}^{(r)}$ of the LCC to write the $u^{(r)}$ in the (trivial) form

$$
u^{(r)}(\boldsymbol{\sigma})=u_{L}^{(r)}(\boldsymbol{\sigma})+\alpha^{(r)}\left(u^{(r)}(\boldsymbol{\sigma})-u_{L}^{(r)}(\boldsymbol{\sigma})\right)+\left(1-\alpha^{(r)}\right)\left(u^{(r)}(\boldsymbol{\sigma})-u_{L}^{(r)}(\boldsymbol{\sigma})\right)
$$

where the $\alpha^{(r)}$ are constant per-phase 'weight factors' between 0 and 1.

Now, as illustrated in Fig. $1(a)$ and $(b)$ for an isotropic material - and in contrast to the functions $u^{(r)}$ and $u_{L}^{(r)}$, which possess only one stationary point (a global minimum)the functions $\Delta u^{(r)}=u^{(r)}-u_{L}^{(r)}$ are non-convex and can exhibit multiple stationary points, including local minima, maxima and saddle points. This suggests that the stationary points of $u^{(r)}$ may be estimated from the stationary points of $u_{L}^{(r)}$, provided that the properties of the linear comparison composite $\mathbb{M}^{(r)}$ and $\gamma^{(r)}$ are selected such that the functions $\Delta u^{(r)}=$ $u^{(r)}-u_{L}^{(r)}$ are also stationary at these points. More specifically, this suggests the following 


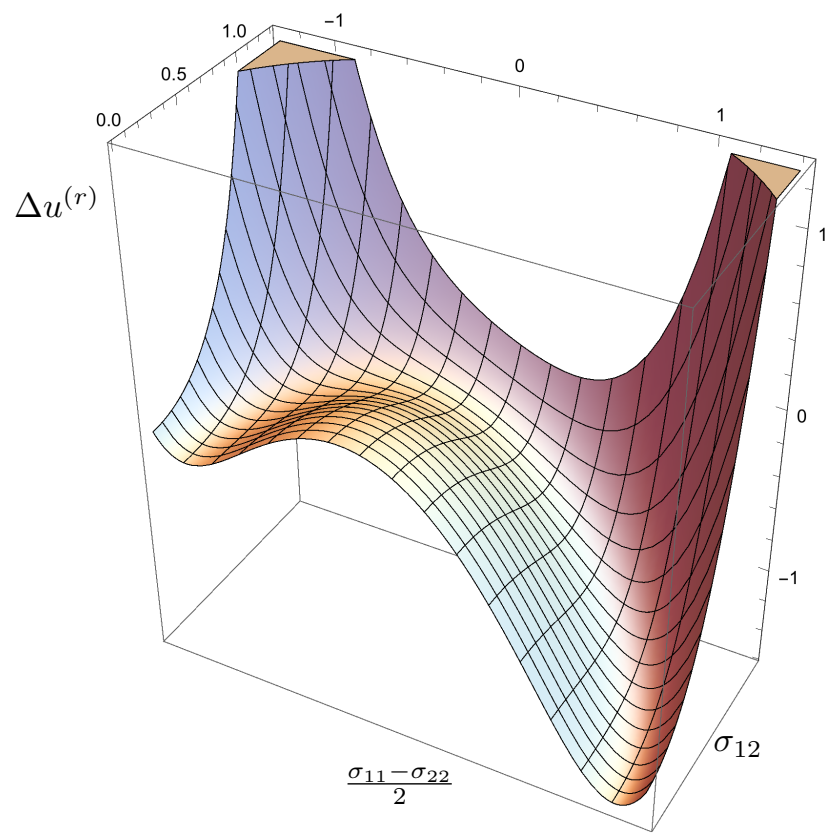

(a)

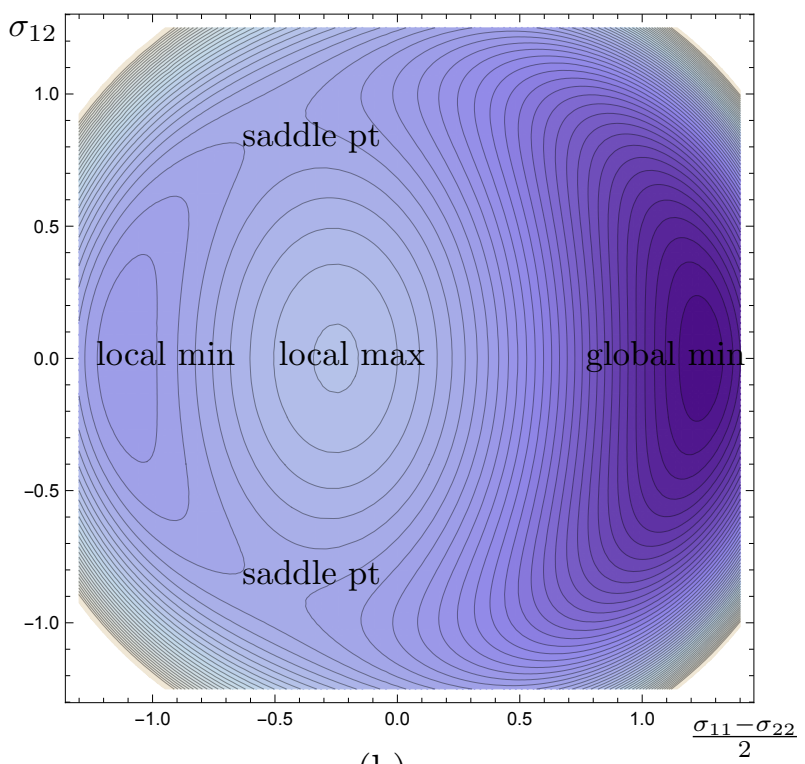

(b)

Figure 1: Two-dimensional example of a function $\Delta u^{(r)}=u^{(r)}-u_{L}^{(r)}$ for an isotropic power-law material with $\gamma^{(r)}=\mathbf{0}$ (for simplicity). (a) 3-D view cut along the $\sigma_{12}=0$ (symmetry plane). (b) 2-D contour plot indicating local maxima, minima and saddle points.

representation for the nonlinear potentials $u^{(r)}$ in terms of the linear comparison potentials $u_{L}^{(r)}$, namely

$$
u^{(r)}(\boldsymbol{\sigma})=\underset{\boldsymbol{\gamma}^{(r)}, \mathbb{M}^{(r)}}{\operatorname{stat}}\left\{u_{L}^{(r)}(\boldsymbol{\sigma})+\alpha^{(r)} \underset{\check{\boldsymbol{\sigma}}^{(r)}}{\operatorname{stat}} \Delta u^{(r)}(\check{\boldsymbol{\sigma}})+\left(1-\alpha^{(r)}\right) \operatorname{stat}_{\hat{\boldsymbol{\sigma}}^{(r)}} \Delta u^{(r)}(\hat{\boldsymbol{\sigma}})\right\},
$$

where use has been made of the symbols $\hat{\boldsymbol{\sigma}}^{(r)}$ and $\check{\boldsymbol{\sigma}}^{(r)}$ to distinguish explicitly between different stationary points of the function $\Delta u^{(r)}=u^{(r)}-u_{L}^{(r)}$. In addition, it should be noted that the representation (21) depends on the specific choice of the (multiple) stationary points of the function $\Delta u^{(r)}=u^{(r)}-u_{L}^{(r)}$, and is therefore not unique in general.

To confirm the validity of this representation for a given selection of stationary points, it is useful to spell out the stationarity conditions involved. Thus, the 'inner' stationary conditions for the variables $\check{\boldsymbol{\sigma}}^{(r)}$ and $\hat{\boldsymbol{\sigma}}^{(r)}$ can be written in the form

$$
\partial_{\boldsymbol{\sigma}} u^{(r)}\left(\hat{\boldsymbol{\sigma}}^{(r)}\right)-\mathbb{M}^{(r)} \hat{\boldsymbol{\sigma}}^{(r)}=\boldsymbol{\gamma}^{(r)}=\partial_{\boldsymbol{\sigma}} u^{(r)}\left(\check{\boldsymbol{\sigma}}^{(r)}\right)-\mathbb{M}^{(r)} \check{\boldsymbol{\sigma}}^{(r)},
$$

where we have used the short-hand notation $\partial_{\boldsymbol{\sigma}}=\partial / \partial \boldsymbol{\sigma}$. Note that these two conditions 
imply the 'generalized secant' condition (Ponte Castañeda, 2002a)

$$
\partial_{\boldsymbol{\sigma}} u^{(r)}\left(\hat{\boldsymbol{\sigma}}^{(r)}\right)-\partial_{\boldsymbol{\sigma}} u^{(r)}\left(\check{\boldsymbol{\sigma}}^{(r)}\right)=\mathbb{M}^{(r)}\left(\hat{\boldsymbol{\sigma}}^{(r)}-\check{\boldsymbol{\sigma}}^{(r)}\right)
$$

On the other hand, the 'outer' stationary conditions for the variables $\mathbb{M}^{(r)}$ and $\gamma^{(r)}$ in expression (21) are given by

$$
\boldsymbol{\sigma}=\alpha^{(r)} \check{\boldsymbol{\sigma}}^{(r)}+\left(1-\alpha^{(r)}\right) \hat{\boldsymbol{\sigma}}^{(r)}
$$

and

$$
\boldsymbol{\sigma} \otimes \boldsymbol{\sigma}=\alpha^{(r)} \check{\boldsymbol{\sigma}}^{(r)} \otimes \check{\boldsymbol{\sigma}}^{(r)}+\left(1-\alpha^{(r)}\right) \hat{\boldsymbol{\sigma}}^{(r)} \otimes \hat{\boldsymbol{\sigma}}^{(r)}
$$

Combining these two expressions leads to the result that

$$
\alpha^{(r)}\left(1-\alpha^{(r)}\right)\left[\left(\hat{\boldsymbol{\sigma}}^{(r)}-\check{\boldsymbol{\sigma}}^{(r)}\right) \otimes\left(\hat{\boldsymbol{\sigma}}^{(r)}-\check{\boldsymbol{\sigma}}^{(r)}\right)\right]=\mathbb{O}
$$

which, together with expression (24), implies that $\check{\boldsymbol{\sigma}}^{(r)}=\boldsymbol{\sigma}=\hat{\boldsymbol{\sigma}}^{(r)}$. In turn, it follows from this observation that equality indeed holds in expression (21).

In addition, it follows by taking the derivative of the right-hand side of expression (21) that

$$
\partial_{\boldsymbol{\sigma}}\{\mathrm{RHS}\}=\partial_{\boldsymbol{\sigma}} u_{L}^{(r)}(\boldsymbol{\sigma})=\boldsymbol{\gamma}^{(r)}+\mathbb{M}^{(r)} \boldsymbol{\sigma}
$$

where we have used the stationarity of the right-hand side of the expression with respect to $\hat{\boldsymbol{\sigma}}^{(r)}$ and $\check{\boldsymbol{\sigma}}^{(r)}$ to obtain the first equality, and the chain rule and stationarity with respect to $\mathbb{M}^{(r)}$ and $\gamma^{(r)}$ to obtain the second equality. Now, it follows from the above-shown result that $\check{\boldsymbol{\sigma}}^{(r)}=\boldsymbol{\sigma}=\hat{\boldsymbol{\sigma}}^{(r)}$, together with expression (22), that $\boldsymbol{\gamma}^{(r)}+\mathbb{M}^{(r)} \boldsymbol{\sigma}=\partial_{\boldsymbol{\sigma}} u^{(r)}(\boldsymbol{\sigma})$, so that, indeed, not only are the values of both sides of the representation (21) identical, but also their derivatives. In particular, as already anticipated, the two sides of the expression (21) have identical stationary points, and therefore the representation (21) for the local potentials $u^{(r)}$ of the nonlinear composite can be used in expression (11) for the macroscopic potential $\widetilde{U}$ of the nonlinear composite. In this way a variational representation will be generated for $\widetilde{U}$ involving optimization of the properties of an LCC with properties defined by the set of variables $\mathbb{M}^{(s)}$ and $\gamma^{(s)}(s=1, . ., N)$. However, as will be seen, the optimality conditions for $\mathbb{M}^{(s)}$ and $\gamma^{(s)}$ will be different from the ones discussed in the context of the local potentials, and will involve the first and second moments of the stress fields in the phases of the LCC. 
Next, to make our expressions more compact, we introduce the 'error' functions

$$
\check{V}^{(r)}\left(\mathbb{M}^{(r)}, \boldsymbol{\gamma}^{(r)}\right)=-\operatorname{stat}_{\check{\boldsymbol{\sigma}}^{(r)}} \Delta u^{(r)}\left(\check{\boldsymbol{\sigma}}^{(r)}\right), \quad \text { and } \quad \hat{V}^{(r)}\left(\mathbb{M}^{(r)}, \boldsymbol{\gamma}^{(r)}\right)=-\operatorname{stat}_{\hat{\boldsymbol{\sigma}}^{(r)}} \Delta u^{(r)}\left(\hat{\boldsymbol{\sigma}}^{(r)}\right)
$$

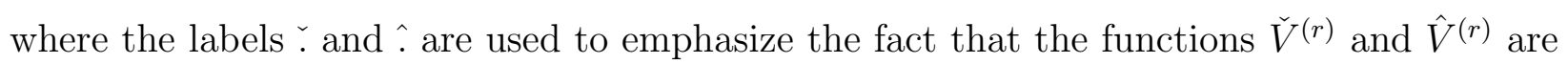
evaluated at different stationary points $\check{\boldsymbol{\sigma}}^{(r)}$ and $\hat{\boldsymbol{\sigma}}^{(r)}$, and are therefore different functions of $\mathbb{M}^{(r)}$ and $\gamma^{(r)}$. (Note that the negative signs have been introduced for consistency with the notations used by Ponte Castañeda, 2002a). It then follows that expression (21) can be rewritten as

$$
u^{(r)}(\boldsymbol{\sigma})=\operatorname{stat}_{\gamma^{(r)}, \mathbb{M}^{(r)}}\left\{\frac{1}{2} \boldsymbol{\sigma} \cdot \mathbb{M}^{(r)} \boldsymbol{\sigma}+\boldsymbol{\gamma}^{(r)} \cdot \boldsymbol{\sigma}-V^{(r)}\left(\mathbb{M}^{(r)}, \boldsymbol{\gamma}^{(r)}\right)\right\}
$$

where the function $u_{L}^{(r)}$ has been written explicitly to display its dependence on $\mathbb{M}^{(r)}$ and $\gamma^{(r)}$, and where the (further compacted) 'error' function $V^{(r)}$ is defined as

$$
V^{(r)}\left(\mathbb{M}^{(r)}, \gamma^{(r)}\right)=\alpha^{(r)} \check{V}^{(r)}\left(\mathbb{M}^{(r)}, \gamma^{(r)}\right)+\left(1-\alpha^{(r)}\right) \hat{V}^{(r)}\left(\mathbb{M}^{(r)}, \gamma^{(r)}\right)
$$

Then, substituting expression (29) for the local potentials $u^{(r)}$ of the nonlinear composite into expression (11) for its macroscopic potential $\widetilde{U}$, and taking the stat over the variables $\mathbb{M}^{(s)}$ and $\gamma^{(s)}(s=1, \ldots, N)$ out of the integral (implied by the angular brackets), it follows that

$$
\widetilde{U}(\overline{\boldsymbol{\sigma}})=\operatorname{stat}_{\boldsymbol{\sigma} \in \mathcal{S}} \operatorname{stat}_{\boldsymbol{\gamma}^{(s)}, \mathbb{M}^{(s)} \in \mathcal{R}}\left\{\sum_{r=1}^{N} c^{(r)}\left\langle\frac{1}{2} \boldsymbol{\sigma} \cdot \mathbb{M}^{(r)} \boldsymbol{\sigma}+\boldsymbol{\gamma}^{(r)} \cdot \boldsymbol{\sigma}-V^{(r)}\left(\mathbb{M}^{(r)}, \boldsymbol{\gamma}^{(r)}\right)\right\rangle^{(r)}\right\},
$$

where it is emphasized that the variables $\mathbb{M}^{(s)}$ and $\gamma^{(s)}(s=1, \ldots, N)$ are now 'trial fields' (i.e., functions of $\mathbf{x}$ ) in some suitable space of functions $\mathcal{R}$. In this context, it should be noted that there are no differential constraints associated with these fields, so that the corresponding Euler-Lagrange conditions are exactly the same as those associated with the local statement (29). Now, at a given stationary point, the order of the stationarity conditions can be interchanged, leading to the result

$$
\widetilde{U}(\overline{\boldsymbol{\sigma}})=\operatorname{stat}_{\boldsymbol{\gamma}^{(s)}, \mathbb{M}^{(s)} \in \mathcal{R}} \operatorname{stat}_{\boldsymbol{\sigma} \in \mathcal{S}}\left\{\sum_{r=1}^{N} c^{(r)}\left\langle\frac{1}{2} \boldsymbol{\sigma} \cdot \mathbb{M}^{(r)} \boldsymbol{\sigma}+\boldsymbol{\gamma}^{(r)} \cdot \boldsymbol{\sigma}-V^{(r)}\left(\mathbb{M}^{(r)}, \boldsymbol{\gamma}^{(r)}\right)\right\rangle^{(r)}\right\}
$$


But the stat over $\boldsymbol{\sigma} \in \mathcal{S}$ only affects the first two terms in the integral, and therefore the variational statement for $\widetilde{U}$ can be rewritten as

$\widetilde{U}(\overline{\boldsymbol{\sigma}})=\operatorname{stat}_{\boldsymbol{\gamma}^{(s)}, \mathbb{M}^{(s)} \in \mathcal{R}}\left\{\operatorname{stat}_{\boldsymbol{\sigma} \in \mathcal{S}} \sum_{r=1}^{N} c^{(r)}\left\langle\frac{1}{2} \boldsymbol{\sigma} \cdot \mathbb{M}^{(r)} \boldsymbol{\sigma}+\boldsymbol{\gamma}^{(r)} \cdot \boldsymbol{\sigma}\right\rangle^{(r)}-\sum_{r=1}^{N} c^{(r)}\left\langle V^{(r)}\left(\mathbb{M}^{(r)}, \boldsymbol{\gamma}^{(r)}\right)\right\rangle^{(r)}\right\}$,

where, once again, it is emphasized that $\mathbb{M}^{(s)}$ and $\gamma^{(s)}$ are fields - and therefore generally non-uniform over the phases of the composite. (It is also for this reason that we have kept the volume averages over the terms involving the error function $V^{(r)}$.)

As a consequence of the non-uniformity of the properties of the phases in the LCC, the variational problem (33) for $\widetilde{U}$ cannot be solved analytically in its full complexity. Therefore, following similar approximations in the earlier work of Ponte Castañeda (2002a), we make use of trial functions $\mathbb{M}^{(s)}$ and $\gamma^{(s)}$ that are (different) constants in the phases $(s=1, \ldots, N)$ of the LCC, leading to the variational estimate

$$
\widetilde{U}(\overline{\boldsymbol{\sigma}}) \approx \widetilde{U}_{N}(\overline{\boldsymbol{\sigma}})=\operatorname{stat}_{\boldsymbol{\gamma}^{(s)}, \mathbb{M}^{(s)}}\left\{\widetilde{U}_{L}(\overline{\boldsymbol{\sigma}})-\sum_{r=1}^{N} c^{(r)} V^{(r)}\left(\mathbb{M}^{(r)}, \boldsymbol{\gamma}^{(r)}\right)\right\}
$$

where it is recalled that both the effective energy $\widetilde{U}_{L}$ of the LCC and the 'error' functions $V^{(r)}$ are functions of the uniform-per-phase properties of the LCC, as determined by $\mathbb{M}^{(s)}$ and $\gamma^{(s)}$. At this point, it should be noted that the new estimate $\widetilde{U}_{N}$ defined by expression (34) for the macroscopic potential $\widetilde{U}$ of the nonlinear composite can be shown to reduce to the stationary variational estimate (49) of Ponte Castañeda (2002a) when the weight factors $\alpha^{(r)}$ in the definition (30) of the function $V^{(r)}$ are all set equal to 0 . In other words, the new stationary estimate (34) provides a generalization of the earlier estimates of Ponte Castañeda (2002a), which essentially consists in the use of the more general error functions $V^{(r)}$ involving multiple stationary point. As will be seen next, the use of such additional error functions will enable the generation of estimates for $\widetilde{U}$ that are stationary with respect to both the variables $\mathbb{M}^{(r)}$ and $\gamma^{(r)}$. This is unlike the corresponding estimates of Ponte Castañeda (2002a), which could only be made stationary with respect to either the $\mathbb{M}^{(r)}$ or the $\gamma^{(r)}$. Loosely speaking, the use of only one error function $V^{(r)}$ in the earlier estimate of Ponte Castañeda (2002a) had the implication that the optimization conditions for the variables $\mathbb{M}^{(r)}$ and $\gamma^{(r)}$ led to conflicting requirements for the variables $\hat{\boldsymbol{\sigma}}^{(r)}$, by identifying them with both the first 
and second moments of the stress in the phases of the LCC-conditions that can not be simultaneously satisfied when fluctuations of the stress field are expected in the phases.

Next, we spell out the stationarity conditions associated with the estimate (34). We begin by noting that the stationary conditions for the 'inner' problems for the variables $\check{\boldsymbol{\sigma}}^{(r)}$ and $\hat{\boldsymbol{\sigma}}^{(r)}$, implicit in the definitions (28) for the functions $\check{V}^{(r)}$ and $\hat{V}^{(r)}$, are precisely the same as for the corresponding phase potentials, namely, equations (22).

Then, using the fact that the stress field in the linear comparison problem is a stationary point of the functional defined by $\widetilde{U}_{L}$, it is found, by means of the chain rule, that the stationarity condition with respect to the eigenstrain variables $\gamma^{(r)}$ can be written as

$$
\overline{\boldsymbol{\sigma}}_{L}^{(r)}=\alpha^{(r)} \check{\boldsymbol{\sigma}}^{(r)}+\left(1-\alpha^{(r)}\right) \hat{\boldsymbol{\sigma}}^{(r)}, r=1, \ldots, N
$$

where $\overline{\boldsymbol{\sigma}}_{L}^{(r)}=\langle\boldsymbol{\sigma}\rangle^{(r)}$ is the average of the stress field in phase $r$ in the problem (17) for the LCC, which may be computed in terms of $\widetilde{U}_{L}$ via the identity $(19)_{1}$. Also, as a consequence, the quantities $\overline{\boldsymbol{\sigma}}_{L}^{(r)}$ are functions of the properties of the LCC, as determined by the variables $\mathbb{M}^{(s)}$ and $\gamma^{(s)}(s=1, \ldots, N$.). Similarly, it is found that the stationarity conditions with respect to the compliance variables $\mathbb{M}^{(r)}$ can be written as

$$
\langle\boldsymbol{\sigma} \otimes \boldsymbol{\sigma}\rangle_{L}^{(r)}=\alpha^{(r)} \check{\boldsymbol{\sigma}}^{(r)} \otimes \check{\boldsymbol{\sigma}}^{(r)}+\left(1-\alpha^{(r)}\right) \hat{\boldsymbol{\sigma}}^{(r)} \otimes \hat{\boldsymbol{\sigma}}^{(r)}, r=1, \ldots, N
$$

where, again, it is emphasized that the variable $\langle\boldsymbol{\sigma} \otimes \boldsymbol{\sigma}\rangle_{L}^{(r)}$ corresponds to the second moment of the stress field in phase $r$ of the LCC, which may be computed via identity $(19)_{2}$, and is therefore also a function of all the variables $\mathbb{M}^{(s)}$ and $\gamma^{(s)}(s=1, \ldots, N$.). Thus, it can be seen that the introduction of the two error functions evaluated at two different stationary points $\check{\boldsymbol{\sigma}}^{(r)}$ and $\hat{\boldsymbol{\sigma}}^{(r)}$ provides in principle the capability to simultaneously satisfy conditions on the first and second moments of the stress fields - something that could not be achieved with the use use of a single stationary point, as first attempted in Ponte Castañeda (2002a).

However, it should be noted that straightforward algebra shows that the conditions (35) and (36) can be combined to obtain the following result for the covariance tensors of the stress fluctuation in the phases

$$
\mathbb{C}_{\boldsymbol{\sigma}}^{(r)}=\alpha^{(r)}\left(1-\alpha^{(r)}\right)\left[\left(\hat{\boldsymbol{\sigma}}^{(r)}-\check{\boldsymbol{\sigma}}^{(r)}\right) \otimes\left(\hat{\boldsymbol{\sigma}}^{(r)}-\check{\boldsymbol{\sigma}}^{(r)}\right)\right], r=1, \ldots, N
$$


as defined by expressions (13). Now, the right-hand side is a rank-1 fourth-order tensor, while the left-hand side is expected to be a full-rank tensor in general. As a consequence, the estimates (34) would not be expected to be fully stationary, in general, and they will need to be generalized further, as discussed in the next subsection. Progress has been made thus far, but additional refinements are needed to achieve full stationarity.

\subsection{Fully stationary variational estimates}

In fact, as illustrated in Fig. 1, the stationary points of the functions $\Delta u^{(r)}=u^{(r)}-u_{L}^{(r)}$ used in the definition of the errors $V^{(r)}$ usually appear in pairs (or groups) due to the symmetries of the strain potentials $u^{(r)}$. In order to be able to account for these symmetryrelated stationary points we introduce here a slight generalization of the LCC consisting in the use of several eigenstrains per phase $\gamma_{(p)}^{(r)}$ (in addition to the compliances $\mathbb{M}^{(r)}$, as before), such that

$$
\gamma^{(r)}=\sum_{p=1}^{M} \beta_{(p)}^{(r)} \gamma_{(p)}^{(r)}
$$

where the $\beta_{(p)}^{(r)}(p=1, \ldots, M)$ are weight factors, such that $0<\beta_{(p)}^{(r)}<1$ and $\sum_{p=1}^{M} \beta_{(p)}^{(r)}=1$, corresponding to each phase $(r=1, \ldots, N)$. Thus, we redefine the strain potential of the LCC via the expressions

$$
u_{L}^{(r)}(\boldsymbol{\sigma})=\frac{1}{2} \boldsymbol{\sigma} \cdot \mathbb{M}^{(r)} \boldsymbol{\sigma}+\sum_{p=1}^{M} \beta_{(p)}^{(r)} \boldsymbol{\gamma}_{(p)}^{(r)} \cdot \boldsymbol{\sigma}
$$

We also redefine the corresponding error functions, so that

$$
\check{V}_{(p)}^{(r)}\left(\mathbb{M}^{(r)}, \boldsymbol{\gamma}_{(p)}^{(r)}\right)=\operatorname{stat}_{\check{\boldsymbol{\sigma}}_{(p)}^{(r)}}\left[\frac{1}{2} \check{\boldsymbol{\sigma}}_{(p)}^{(r)} \cdot \mathbb{M}^{(r)} \check{\boldsymbol{\sigma}}_{(p)}^{(r)}+\boldsymbol{\gamma}_{(p)}^{(r)} \cdot \check{\boldsymbol{\sigma}}_{(p)}^{(r)}-u^{(r)}\left(\check{\boldsymbol{\sigma}}_{(p)}^{(r)}\right)\right]
$$

and similarly for $\hat{V}_{(p)}^{(r)}\left(\mathbb{M}^{(r)}, \gamma_{(p)}^{(r)}\right)$, where, once again, it is emphasized that the functions $\check{V}_{(p)}^{(r)}$ and $\hat{V}_{(p)}^{(r)}$ (for fixed $r$ and $p$ ) are evaluated at different stationary points $\check{\boldsymbol{\sigma}}_{(p)}^{(r)}$ and $\hat{\boldsymbol{\sigma}}_{(p)}^{(r)}$, respectively. As will be shown more precisely in the example in section 6 , the $M$ different stationary points $\check{\boldsymbol{\sigma}}_{(p)}^{(r)}(p=1, \ldots, M$, for fixed phase $r)$ are related by symmetry, and similarly for the $M$ stationary points $\hat{\boldsymbol{\sigma}}_{(p)}^{(r)}$. For example, two of them may be mirror images of each other about a symmetry axis that is determined either by the loading condition, or by the material properties. 
We can then write the corresponding generalization of the variational estimate (34) for the macroscopic potential of the nonlinear composite as

$$
\widetilde{U}(\overline{\boldsymbol{\sigma}}) \approx \widetilde{U}_{N}(\overline{\boldsymbol{\sigma}})=\operatorname{stat}_{\substack{(s) \\ \gamma_{(p)}, \mathbb{M}^{(s)}}}\left\{\widetilde{U}_{L}(\overline{\boldsymbol{\sigma}})-\sum_{r=1}^{N} c^{(r)} \sum_{p=1}^{M} \beta_{(p)}^{(r)} V_{(p)}^{(r)}\left(\mathbb{M}^{(r)}, \gamma_{(p)}^{(r)}\right)\right\},
$$

where $V_{(p)}^{(r)}$ is the compacted error function

$$
V_{(p)}^{(r)}\left(\mathbb{M}^{(r)}, \gamma_{(p)}^{(r)}\right)=\alpha^{(r)} \check{V}_{(p)}^{(r)}\left(\mathbb{M}^{(r)}, \gamma_{(p)}^{(r)}\right)+\left(1-\alpha^{(r)}\right) \hat{V}_{(p)}^{(r)}\left(\mathbb{M}^{(r)}, \boldsymbol{\gamma}_{(p)}^{(r)}\right)
$$

and $\widetilde{U}_{L}$ is the macroscopic potential of the LCC, as defined by expression (17), but now associated with the phase potentials (39). It is therefore also a function of the variables $\mathbb{M}^{(r)}$ and $\gamma_{(p)}^{(r)}$.

Then, the stationarity conditions associated with the functions $\check{V}_{(p)}^{(r)}$ and $\hat{V}_{(p)}^{(r)}$ in the estimate estimate (41), as determined by expressions (40), are simply given by

$$
\partial_{\boldsymbol{\sigma}} u^{(r)}\left(\hat{\boldsymbol{\sigma}}_{(p)}^{(r)}\right)-\mathbb{M}^{(r)} \hat{\boldsymbol{\sigma}}_{(p)}^{(r)}=\boldsymbol{\gamma}_{(p)}^{(r)}=\partial_{\boldsymbol{\sigma}} u^{(r)}\left(\check{\boldsymbol{\sigma}}_{(p)}^{(r)}\right)-\mathbb{M}^{(r)} \check{\boldsymbol{\sigma}}_{(p)}^{(r)}, r=1, \ldots, N
$$

which provide $2 M$ conditions for each phase.

The stationarity conditions with respect to the eigenstrains $\gamma_{(p)}^{(r)}$ provide the $M$ additional equations (for each phase)

$$
\overline{\boldsymbol{\sigma}}_{L}^{(r)}=\alpha^{(r)} \check{\boldsymbol{\sigma}}_{(p)}^{(r)}+\left(1-\alpha^{(r)}\right) \hat{\boldsymbol{\sigma}}_{(p)}^{(r)}, r=1, \ldots, N
$$

where it is recalled that $\overline{\boldsymbol{\sigma}}_{L}^{(r)}$ is the average of the stress field in phase $r$ in the problem (17) for the LCC, which may be computed via identity $(19)_{1}$. As a consequence, $\overline{\boldsymbol{\sigma}}_{L}^{(r)}$ is a function of the properties of the LCC, as determined by $\mathbb{M}^{(s)}$ and $\gamma_{(q)}^{(s)}(s=1, \ldots, N ; q=1, \ldots, M)$, as well as the prescribed macroscopic stress $\overline{\boldsymbol{\sigma}}$. On the other hand, it is found that the stationarity conditions with respect to the compliances $\mathbb{M}^{(r)}$ can be written as

$$
\langle\boldsymbol{\sigma} \otimes \boldsymbol{\sigma}\rangle_{L}^{(r)}=\sum_{p=1}^{M} \beta_{(p)}^{(r)}\left[\alpha^{(r)} \check{\boldsymbol{\sigma}}_{(p)}^{(r)} \otimes \check{\boldsymbol{\sigma}}_{(p)}^{(r)}+\left(1-\alpha^{(r)}\right) \hat{\boldsymbol{\sigma}}_{(p)}^{(r)} \otimes \hat{\boldsymbol{\sigma}}_{(p)}^{(r)}\right], r=1, \ldots, N
$$

where $\langle\boldsymbol{\sigma} \otimes \boldsymbol{\sigma}\rangle_{L}^{(r)}$ denotes the second moment of the stress field in phase $r$ of the LCC, and may be computed via identity $(19)_{2}$ in terms of all the variables $\mathbb{M}^{(s)}$ and $\boldsymbol{\gamma}_{(q)}^{(s)}$, as well as the macroscopic stress $\overline{\boldsymbol{\sigma}}$. 
Finally, it can be shown that the conditions (44) and (45) can be combined to obtain the following result for the covariance tensors of the stress fluctuation in the phases of the LCC, namely

$$
\mathbb{C}_{\boldsymbol{\sigma}}^{(r)}=\alpha^{(r)}\left(1-\alpha^{(r)}\right) \sum_{p=1}^{M} \beta_{(p)}^{(r)}\left[\left(\hat{\boldsymbol{\sigma}}_{(p)}^{(r)}-\check{\boldsymbol{\sigma}}_{(p)}^{(r)}\right) \otimes\left(\hat{\boldsymbol{\sigma}}_{(p)}^{(r)}-\check{\boldsymbol{\sigma}}_{(p)}^{(r)}\right)\right], r=1, \ldots, N
$$

It is remarked that the right-hand side can now, at least in principle, be a fourth-order tensor of full rank, consistent with the left-hand side $\mathbb{C}_{\boldsymbol{\sigma}}^{(r)}$, which is expected to be a fullrank tensor in general. Thus, $M$ should be taken to be sufficiently large to ensure that the right-hand side is of full rank. This means that, among all possible stationary points of the function $\Delta u^{(r)}$ defining the 'error' functions $\check{V}_{(p)}^{(r)}$ and $\hat{V}_{(p)}^{(r)}$, enough of them should be selected to be sure to satisfy condition (46). In other words, the estimates (41) can be made fully stationary with respect to the variables $\mathbb{M}^{(s)}$ and $\gamma_{(q)}^{(s)}$-under suitable selection of the weights $\alpha^{(r)}$ and $\beta_{(p)}^{(r)}$-for an appropriate number $(2 M)$ of stationary points $\hat{\boldsymbol{\sigma}}_{(p)}^{(r)}$ and $\check{\boldsymbol{\sigma}}_{(p)}^{(r)}$. Of course, this choice will be highly dependent on the specific functional form of the potentials $u^{(r)}$, and it is difficult to make more precise statements without further details on the functions $u^{(r)}$. However, complete details on the selection of the stationary points and of the corresponding weights $\alpha^{(r)}$ and $\beta_{(p)}^{(r)}$ will be provided in the context of an example for two-dimensional porous, isotropic, power-law material in section 6 .

At this point, however, it is interesting to note that expression (44) can be rewritten as

$$
\hat{\boldsymbol{\sigma}}_{(p)}^{(r)}-\check{\boldsymbol{\sigma}}_{(p)}^{(r)}=\frac{1}{\alpha^{(r)}}\left(\hat{\boldsymbol{\sigma}}_{(p)}^{(r)}-\overline{\boldsymbol{\sigma}}_{L}^{(r)}\right)=\frac{1}{1-\alpha^{(r)}}\left(\overline{\boldsymbol{\sigma}}_{L}^{(r)}-\check{\boldsymbol{\sigma}}_{(p)}^{(r)}\right)
$$

while expression (46) can be rewritten as

$$
\begin{aligned}
\mathbb{C}_{\boldsymbol{\sigma}}^{(r)} & =\frac{1-\alpha^{(r)}}{\alpha^{(r)}} \sum_{p=1}^{M} \beta_{(p)}^{(r)}\left[\left(\hat{\boldsymbol{\sigma}}_{(p)}^{(r)}-\overline{\boldsymbol{\sigma}}_{L}^{(r)}\right) \otimes\left(\hat{\boldsymbol{\sigma}}_{(p)}^{(r)}-\overline{\boldsymbol{\sigma}}_{L}^{(r)}\right)\right] \\
& =\frac{\alpha^{(r)}}{1-\alpha^{(r)}} \sum_{p=1}^{M} \beta_{(p)}^{(r)}\left[\left(\overline{\boldsymbol{\sigma}}_{L}^{(r)}-\check{\boldsymbol{\sigma}}_{(p)}^{(r)}\right) \otimes\left(\overline{\boldsymbol{\sigma}}_{L}^{(r)}-\check{\boldsymbol{\sigma}}_{(p)}^{(r)}\right)\right] .
\end{aligned}
$$

It becomes clear from this form of the equations that, when the $\alpha^{(r)}$ are set equal to $1 / 2$, the stationary points $\hat{\boldsymbol{\sigma}}_{(p)}^{(r)}$ and $\check{\boldsymbol{\sigma}}_{(p)}^{(r)}$ become symmetrically distributed about the phase averages $\overline{\boldsymbol{\sigma}}_{L}^{(r)}$ (i.e., $\hat{\boldsymbol{\sigma}}_{(p)}^{(r)}-\overline{\boldsymbol{\sigma}}_{L}^{(r)}=\overline{\boldsymbol{\sigma}}_{L}^{(r)}-\check{\boldsymbol{\sigma}}_{(p)}^{(r)}$ ), with the covariance tensor $\mathbb{C}_{\boldsymbol{\sigma}}^{(r)}$ determining their 'distance' from the phase average $\overline{\boldsymbol{\sigma}}_{L}^{(r)}$. Although the estimates (41) are fully stationary with respect to the variables $\mathbb{M}^{(r)}$ and $\gamma_{(r)}^{(s)}$ independently of the value of the weight factors $\alpha^{(r)}$, and other 
choices could indeed be envisaged for these parameters, in the applications to be considered in section 6, use will be made of the "symmetric" prescription $\alpha^{(r)}=1 / 2$.

The stationarity conditions (43), (44) and (45) (or, alternatively, (43), (47) and (48)) provide, at least in principle, a sufficient number of equations to solve for the $3 N \times M+N$ unknown variables $\check{\boldsymbol{\sigma}}_{(p)}^{(r)}, \hat{\boldsymbol{\sigma}}_{(p)}^{(r)}, \mathbb{M}^{(r)}$ and $\boldsymbol{\gamma}_{(p)}^{(r)}$ (for an appropriate choice of the weight factors $\alpha^{(r)}$ and $\left.\beta_{(p)}^{(r)}\right)$. In this context, it should also be noted that the selection of the weight factors $\beta_{(p)}^{(r)}$ will be dictated by appropriate symmetry requirements as will be discussed in the context of a specific example in section 6 . In any event, the solutions of these equations can then be used to generate fully stationary variational estimates for $\widetilde{U}$ via expression (41).

\subsection{Additional properties of the stationary variational estimates}

Simplified expression for the macroscopic strain potential. The new variational estimates (41) exhibit several important properties that will prove to be useful in applications. The first is a consequence of the full stationarity of these estimates with respect to the variables $\check{\boldsymbol{\sigma}}_{(p)}^{(r)}$, $\hat{\boldsymbol{\sigma}}_{(p)}^{(r)}, \mathbb{M}^{(r)}$ and $\gamma_{(p)}^{(r)}$, which can be used directly in expression (41) to obtain the simplified result

$$
\widetilde{U}_{N}(\overline{\boldsymbol{\sigma}})=\sum_{r=1}^{N} c^{(r)} \sum_{p=1}^{M} \beta_{(p)}^{(r)}\left[\alpha^{(r)} u^{(r)}\left(\check{\boldsymbol{\sigma}}_{(p)}^{(r)}\right)+\left(1-\alpha^{(r)}\right) u^{(r)}\left(\hat{\boldsymbol{\sigma}}_{(p)}^{(r)}\right)\right] .
$$

Thus, it can be seen that the final result for the effective potential $\widetilde{U}$ of the nonlinear composite involves a weighted average of the stationary points $\check{\boldsymbol{\sigma}}_{(p)}^{(r)}$ and $\hat{\boldsymbol{\sigma}}_{(p)}^{(r)}$, which as we have seen (see eqns. (44) and (45)) depend on the first and second moments of the stress field in the phases of the LCC. As such, expression (49) provides a generalization of the 'variational' bounds of Ponte Castañeda (1991) (see also Suquet, 1995; Ponte Castañeda and Suquet, 1998; Idiart and Ponte Castañeda, 2007b), which could be expressed in terms of the (single) minimum stationary point (in each phase), in turn associated to the second moments of the fields of the phases in a simplified LCC depending only on compliances $\mathbb{M}^{(r)}$ (but not eigenstrains $\gamma_{(p)}^{(r)}$ ). In addition, it provides a generalization of the 'second-order' estimates of Ponte Castañeda (1996) (see also Ponte Castañeda and Willis, 1999), which also made use of a single stationary point (per phase), albeit related in this case to the first moments of the fields in the phases of the LCC. As already mentioned, the new estimates (41) also provide a generalization of the estimates of Ponte Castañeda (2002a), which was a 
first attempt to generate nonlinear estimates incorporating dependence on both the first and second moments of the fields in the phases of the LCC. However, the estimates (41) - unlike the earlier ones - generate this dependence on the first and second moments of the fields in a way that is fully consistent with a stationary variational principle.

Macroscopic stress-strain relation via the $L C C$. The new variational estimates (41) exhibit several important properties that will prove to be very useful in applications. The first is a consequence of the full stationarity of these estimates with respect to the variables $\check{\boldsymbol{\sigma}}_{(p)}^{(r)}, \hat{\boldsymbol{\sigma}}_{(p)}^{(r)}$, $\mathbb{M}^{(r)}$ and $\gamma_{(p)}^{(r)}$, as well as of the fact that the stress field in the linear comparison problem is a minimum (and therefore also a stationary point of the functional $\widetilde{U}_{L}$ ). Indeed, by means of the chain rule (see, for example, Idiart and Ponte Castañeda, 2007a), it is easy to show that the macroscopic stress-strain relation for the nonlinear composite, as determined by relation (10), reduces to the corresponding stress-strain relation for the LCC, where the variables $\mathbb{M}^{(r)}$ and $\gamma_{(p)}^{(r)}$ are, of course, still determined by the stationarity conditions of the previous section, and are functions of the macroscopic stress $\overline{\boldsymbol{\sigma}}$ (see also deBotton and Ponte Castañeda, 1993, for an earlier result in the context of the 'variational' approach). Thus, making use of expression (18) for $\widetilde{U}_{L}$, and of the results of Appendix A, including expression (A.4) for the corresponding macroscopic stress-strain relation, we have that

$$
\overline{\boldsymbol{\epsilon}}=\tilde{\mathbb{M}} \overline{\boldsymbol{\sigma}}+\tilde{\gamma}
$$

where $\widetilde{\mathbb{M}}$ and $\tilde{\gamma}$ are the effective compliance and 'thermal strain' (or eigenstrain) of the LCC, respectively (recall that the $\boldsymbol{\gamma}^{(r)}$ are given in terms of the $\boldsymbol{\gamma}_{(p)}^{(r)}$, via expression (38)). These quantities can be expressed in terms of the corresponding stress concentration tensors $\mathbb{B}^{(r)}$ and $\mathbf{b}^{(r)}$, as defined by expressions (A.7), via expressions (A.8). Of course, specific estimates for the concentration tensors $\mathbb{B}^{(r)}$ and $\mathbf{b}^{(r)}$, in terms of the properties of the LCC, will depend on the microstructure of the composite (Willis, 1981). The interest in the property (50) of the fully stationary estimates lies in the fact that this expression is much easier to evaluate than the derivative of expression (49) for the effective potential $\widetilde{U}$, since the effective potential $\widetilde{U}_{L}$ of the LCC, as defined by expression (18), already requires the computation of $\widetilde{\mathbb{M}}$ and $\tilde{\gamma}$. This simplification is especially significant for problems with a large number of phases, such as polycrystals (Liu and Ponte Castañeda, 2004; Liu et al., 2005). 
Exactness to second-order in the heterogeneity contrast. The prior property essentially shows that the fully stationary estimate is exact to second-order in the contrast, provided that the corresponding estimate for the LCC also be exact to second-order in the contrast. This basically follows from the fact that, for small contrast, the phases averages and second moments correspond to the macroscopic average, and therefore the moduli of the LCC correspond to the tangent approximation, which is already known (e.g., Ponte Castañeda, 1996) to reproduce the estimates of Suquet and Ponte Castañeda (1993) exactly to second order in the contrast.

Field statistics for the nonlinear composite via the LCC. Idiart and Ponte Castañeda (2007b) developed a general procedure for computing the moments of the stress and strain field in the phases of the nonlinear composite by means of suitably perturbed nonlinear problems.

Thus, for example, the first moment, or average, of the stress field in phase $r$ of the nonlinear composite may be obtained via the identity (Proposition 3.1)

$$
\overline{\boldsymbol{\sigma}}^{(r)}=\left.\frac{1}{c^{(r)}} \partial_{\boldsymbol{\eta}^{(r)}} \widetilde{U}_{\boldsymbol{\eta}}\right|_{\boldsymbol{\eta}^{(r)}=\mathbf{0}}
$$

where $\boldsymbol{\eta}^{(r)}$ is a constant, symmetric, second-order tensor, and $\widetilde{U}_{\boldsymbol{\eta}}$ denotes the effective potential of a composite with (perturbed) local potential

$$
u_{\boldsymbol{\eta}}(\mathbf{x}, \boldsymbol{\sigma})=\sum_{s=1}^{N} \chi^{(s)}(\mathbf{x}) u^{(s)}(\boldsymbol{\sigma})+\chi^{(r)}(\mathbf{x}) \boldsymbol{\eta}^{(r)} \cdot \boldsymbol{\sigma} .
$$

(In this last expression, the $u^{(s)}$ are the strain potentials of the actual nonlinear composite.) In particular, this result - when applied to the LCC, as defined by expressions (17) and (39) -implies that

$$
\overline{\boldsymbol{\sigma}}_{L}^{(r)}=\left.\frac{1}{c^{(r)}} \partial_{\boldsymbol{\eta}^{(r)}} \widetilde{U}_{L \boldsymbol{\eta}}\right|_{\boldsymbol{\eta}^{(r)}=\mathbf{0}}
$$

where $\widetilde{U}_{L \boldsymbol{\eta}}$ denotes the effective potential of a composite with (perturbed) local potential

$$
u_{L \boldsymbol{\eta}}(\mathbf{x}, \boldsymbol{\sigma})=\sum_{s=1}^{N} \chi^{(s)}(\mathbf{x}) u_{L}^{(s)}(\boldsymbol{\sigma})+\chi^{(r)}(\mathbf{x}) \boldsymbol{\eta}^{(r)} \cdot \boldsymbol{\sigma}
$$

(The result (53) is, of course, fully consistent with the special result (19) for linear thermoelastic composites.) Then, noting that the corresponding macroscopic potential for the 
perturbed nonlinear composite may be estimated as

$$
\widetilde{U}_{\boldsymbol{\eta}}(\overline{\boldsymbol{\sigma}})=\operatorname{stat}_{\substack{(s) \\ \boldsymbol{\gamma}_{(p)}^{(s)}, \mathbb{M}^{(s)}}}\left\{\widetilde{U}_{L \boldsymbol{\eta}}(\overline{\boldsymbol{\sigma}})-\sum_{r=1}^{N} c^{(r)} \sum_{p=1}^{M} \beta_{(p)}^{(r)} V_{(p)}^{(r)}\left(\mathbb{M}^{(r)}, \boldsymbol{\gamma}_{(p)}^{(r)}\right)\right\}
$$

where the functions $V_{(p)}^{(r)}$, as defined by (42) in terms of $\check{V}_{(p)}^{(r)}$ and $\hat{V}_{(p)}^{(r)}$, are independent of $\boldsymbol{\eta}^{(r)}$, and using the chain rule and the stationarity with respect to the variables $\boldsymbol{\gamma}_{(p)}^{(r)}$ and $\mathbb{M}^{(r)}$, it is easy to see that the quantities $\partial_{\boldsymbol{\eta}^{(r)}} \tilde{U}_{\boldsymbol{\eta}}$ and $\partial_{\boldsymbol{\eta}^{(r)}} \widetilde{U}_{L \boldsymbol{\eta}}$ (evaluated at the optimal values of the $\boldsymbol{\gamma}_{(p)}^{(r)}$ and $\left.\mathbb{M}^{(r)}\right)$ are identical. By setting $\boldsymbol{\eta}^{(r)}=0$, it is concluded that

$$
\overline{\boldsymbol{\sigma}}^{(r)}=\overline{\boldsymbol{\sigma}}_{L}^{(r)} \text {. }
$$

In other words, the phase averages of the stress field in the nonlinear composite can be estimated directly from the phase averages of the stress field in the LCC. Note that these quantities are required anyway for the computation of the fully stationary estimate (41) via the stationarity conditions (44), and therefore no additional calculations are needed to obtain the phase averages of the stress in the nonlinear composite.

Similarly, by Proposition 3.4 of Idiart and Ponte Castañeda (2007b), the first moment, or average, of the strain field in phase $r$ of the nonlinear composite may be obtained via

$$
\overline{\boldsymbol{\epsilon}}^{(r)}=-\left.\frac{1}{c^{(r)}} \partial_{\boldsymbol{\tau}^{(r)}} \widetilde{U}_{\boldsymbol{\tau}}\right|_{\boldsymbol{\tau}^{(r)}=\mathbf{0}},
$$

where $\boldsymbol{\tau}^{(r)}$ is a constant, symmetric, second-order tensor, and $\widetilde{U}_{\boldsymbol{\tau}}$ denotes the effective potential of a composite with (perturbed) local potential given by

$$
u_{\boldsymbol{\tau}}(\mathbf{x}, \boldsymbol{\sigma})=\sum_{\substack{s=1 \\ s \neq r}}^{N} \chi^{(s)}(\mathbf{x}) u^{(s)}(\boldsymbol{\sigma})+\chi^{(r)}(\mathbf{x}) u^{(r)}\left(\boldsymbol{\sigma}-\boldsymbol{\tau}^{(r)}\right) .
$$

(In this last expression, the $u^{(s)}$ are once again the strain potentials of the actual nonlinear composite.) In particular, this result - when applied to the LCC, as defined by expressions (17) and (39) -implies that

$$
\overline{\boldsymbol{\epsilon}}_{L}^{(r)}=-\left.\frac{1}{c^{(r)}} \partial_{\boldsymbol{\tau}^{(r)}} \widetilde{U}_{L \boldsymbol{\tau}}\right|_{\boldsymbol{\tau}^{(r)}=\mathbf{0}}
$$

where $\widetilde{U}_{L \boldsymbol{\tau}}$ denotes the effective potential of a composite with (perturbed) local potential

$$
u_{L \boldsymbol{\eta}}(\mathbf{x}, \boldsymbol{\sigma})=\sum_{\substack{s=1 \\ s \neq r}}^{N} \chi^{(s)}(\mathbf{x}) u_{L}^{(s)}(\boldsymbol{\sigma})+\chi^{(r)}(\mathbf{x}) u_{L}^{(r)}\left(\boldsymbol{\sigma}-\boldsymbol{\tau}^{(r)}\right) .
$$


As was the case for the phases averages of the stress field, it is easy to see that the fully stationary estimate for $\widetilde{U}_{\boldsymbol{\tau}}$ in terms of $\widetilde{U}_{L \boldsymbol{\tau}}$ involves error functions $\check{V}_{(p)}^{(r)}$ and $\hat{V}_{(p)}^{(r)}$, which are independent of the variables $\boldsymbol{\tau}^{(r)}$, and is stationary with respect to the variables $\gamma_{(p)}^{(r)}$ and $\mathbb{M}^{(r)}$, leading to the conclusion - via the chain rule - that $\partial_{\tau}^{(r)} \tilde{U}_{\tau}=\partial_{\tau}^{(r)} \widetilde{U}_{L \tau}$. It follows that

$$
\overline{\boldsymbol{\epsilon}}^{(r)}=\overline{\boldsymbol{\epsilon}}_{L}^{(r)}
$$

Thus, the phase averages of the strain field in the nonlinear composite can also be estimated directly from the corresponding phase averages of the strain field in the LCC. It should be noted in this context that the results (61) for the phase averages of the strain are consistent with the corresponding result (50) for the macroscopic strain, since

$$
\overline{\boldsymbol{\epsilon}}=\sum_{s=1}^{N} c^{(s)} \overline{\boldsymbol{\epsilon}}^{(s)}=\sum_{s=1}^{N} c^{(s)} \overline{\boldsymbol{\epsilon}}_{L}^{(s)}=\tilde{\mathbb{M}} \overline{\boldsymbol{\sigma}}+\tilde{\boldsymbol{\gamma}},
$$

It is also possible to similarly show by means of Corollaries 3.3 and 3.7 of Idiart and Ponte Castañeda (2007b) that the second moments of the stress and strain fields in the nonlinear composite can be consistently estimated from the LCC, that is,

$$
\langle\boldsymbol{\sigma} \otimes \boldsymbol{\sigma}\rangle^{(r)}=\langle\boldsymbol{\sigma} \otimes \boldsymbol{\sigma}\rangle_{L}^{(r)} \quad \text { and } \quad\langle\boldsymbol{\epsilon} \otimes \boldsymbol{\epsilon}\rangle^{(r)}=\langle\boldsymbol{\epsilon} \otimes \boldsymbol{\epsilon}\rangle_{L}^{(r)} .
$$

In summary, the full stationarity of the variational estimate (41) implies that, not only can the macroscopic stress-strain relation for the nonlinear composite be estimated directly from the corresponding stress-strain relation of the LCC, but, in addition, so can the first and second moments of the stress and strain fields in the nonlinear composite. Since the variational estimate (41) already requires the computation of the first and second moments of the stress field in the LCC, the fact that these quantities also provide estimates for the corresponding first and second moments of the stress field in the actual nonlinear composite is very convenient, since the direct computation from the appropriate perturbed potentials is not straightforward, in general. For example, it was shown by Idiart and Ponte Castañeda (2007b) that first and second moments of the stress and strain fields in the earlier versions (Ponte Castañeda, 1996, 2002a) of the 'tangent' and 'generalized secant' second-order estimates - which are either not stationary with respect to the variables $\mathbb{M}^{(r)}$, or $\boldsymbol{\gamma}^{(r)}$, respectively —involved additional terms which were difficult to compute, except for 
certain special choices of these parameters. On the other hand, the present fully stationary version of the second-order estimates is similar to the variational bounds of Ponte Castañeda (1991), for which the first and second moments of the stress and strain fields in the LCC can be used to directly estimate the corresponding moments of the fields in the actual nonlinear composite. This is a feature that certainly makes worthwhile the additional complexities associated with the use of multiple error functions in the new stationary estimates (41).

\section{Duality results}

\subsection{The $L C C$}

Given the definitions (39) and (17) for the local and macroscopic strain potentials, $u_{L}^{(r)}$ and $\widetilde{U}_{L}$, of the LCC, it is easy to compute via the Legendre transformation (4) the corresponding local and macroscopic stress potentials. Thus, making use of the results (A.3) in Appendix A, we have that

$$
w_{L}^{(r)}(\boldsymbol{\epsilon})=\frac{1}{2} \boldsymbol{\epsilon} \cdot \mathbb{L}^{(r)} \boldsymbol{\epsilon}+\boldsymbol{\tau}^{(r)} \cdot \boldsymbol{\epsilon}+\frac{1}{2} \boldsymbol{\tau}^{(r)} \cdot\left(\mathbb{L}^{(r)}\right)^{-1} \boldsymbol{\tau}^{(r)},
$$

where $\mathbb{L}^{(r)}$ is a uniform modulus tensor, and $\boldsymbol{\tau}^{(r)}$ a uniform eigenstress (or 'thermal' stress), corresponding to each of the phases. They are related to the properties of the strain potential $u_{L}^{(r)}$, as given by $\mathbb{M}^{(r)}$ and $\gamma_{(p)}^{(r)}$, via

$$
\mathbb{L}^{(r)}=\left(\mathbb{M}^{(r)}\right)^{-1}, \quad \text { and } \quad \boldsymbol{\tau}^{(r)}=\sum_{p=1}^{M} \beta_{(p)}^{(r)} \boldsymbol{\tau}_{(p)}^{(r)}, \quad \text { with } \quad \boldsymbol{\tau}_{(p)}^{(r)}=-\left(\mathbb{M}^{(r)}\right)^{-1} \boldsymbol{\gamma}_{(p)}^{(r)}
$$

Then, letting the stress potential for the linear comparison composite be

$$
w_{L}(\mathbf{x}, \boldsymbol{\epsilon})=\sum_{r=1}^{N} \chi^{(r)}(\mathbf{x}) w_{L}^{(r)}(\boldsymbol{\epsilon})
$$

we can write the effective, or homogenized stress potential for the composite in the form (Laws, 1973; Willis, 1981)

$$
\widetilde{W}_{L}(\overline{\boldsymbol{\epsilon}})=\min _{\boldsymbol{\epsilon} \in \mathcal{K}}\left\langle w_{L}(\mathbf{x}, \boldsymbol{\epsilon})\right\rangle=\frac{1}{2} \overline{\boldsymbol{\epsilon}} \cdot \widetilde{\mathbb{L}} \overline{\boldsymbol{\epsilon}}+\tilde{\boldsymbol{\tau}} \cdot \overline{\boldsymbol{\epsilon}}+\tilde{f}
$$

where $\tilde{\mathbb{L}}, \tilde{\boldsymbol{\tau}}$ and $\tilde{f}$ are the effective modulus, effective eigenstress and effective energy at zero strain. They are related to the effective compliance $\widetilde{\mathbb{M}}$, effective eigenstrain $\tilde{\gamma}$ and effective energy at zero stress $\tilde{g}$ via relations (A.9). As already mentioned in the context of the 
problem for $\widetilde{U}_{L}$, the values of these effective variables will depend not only on the properties of the phases, but also on the specific microstructure. It should also be emphasized that the potentials $w_{L}^{(r)}$, as well as the effective potential $\widetilde{W}_{L}$, as given by expressions (64) and (67), contain some extra constant terms (depending on the eigenstresses $\boldsymbol{\tau}^{(r)}$ and moduli $\mathbb{L}^{(r)}$ ), which are not present in the corresponding strain potentials $u_{L}^{(r)}$ and $\widetilde{U}_{L}$. As will be seen next, such terms are necessary for the full stationarity of the dual version of the problem for the nonlinear composite.

\subsection{The nonlinear composite}

To generate an estimate for the effective stress potential $\widetilde{W}$ of the nonlinear composite, we will need to compute the Legendre transform of expression (41) for $\widetilde{U}$. However, before doing so, it will be useful to rewrite the nonlinear error functions $\check{V}_{(p)}^{(r)}$ and $\hat{V}_{(p)}^{(r)}$ in terms of the dual variables $\mathbb{L}^{(r)}$ and $\boldsymbol{\tau}^{(r)}$. Thus, we note that we can write the functions $\check{V}_{(p)}^{(r)}$-and similarly for the functions $\hat{V}_{(p)}^{(r)}$-in the form

$$
\check{V}_{(p)}^{(r)}\left(\mathbb{M}^{(r)}, \gamma_{(p)}^{(r)}\right)=\underset{\check{\boldsymbol{\sigma}}_{(p)}^{(r)}}{\operatorname{stat}}\left[u_{L}^{(r)}\left(\check{\boldsymbol{\sigma}}_{(p)}^{(r)}\right)-u^{(r)}\left(\check{\boldsymbol{\sigma}}_{(p)}^{(r)}\right)\right]
$$

where

$$
u_{L(p)}^{(r)}(\check{\boldsymbol{\sigma}})=\frac{1}{2} \check{\boldsymbol{\sigma}} \cdot \mathbb{M}^{(r)} \check{\boldsymbol{\sigma}}+\boldsymbol{\gamma}_{(p)}^{(r)} \cdot \check{\boldsymbol{\sigma}}
$$

Then, defining the Legendre transform of $u_{L}^{(p)}(r)$ via

$$
w_{L}^{(r)}(\boldsymbol{\epsilon})=\frac{1}{2} \boldsymbol{\epsilon} \cdot \mathbb{L}^{(r)} \boldsymbol{\epsilon}+\boldsymbol{\tau}_{(r)}^{(p)} \cdot \boldsymbol{\epsilon}+\frac{1}{2} \boldsymbol{\tau}_{(p)}^{(r)} \cdot\left(\mathbb{L}^{(r)}\right)^{-1} \boldsymbol{\tau}_{(p)}^{(r)}
$$

where the $\mathbb{L}^{(r)}$ and $\boldsymbol{\tau}_{(p)}^{(r)}$ are given in terms of the $\mathbb{M}^{(r)}$ and $\boldsymbol{\gamma}_{(p)}^{(r)}$ by the same expressions (65) as for the LCC, it follows that

$$
\begin{aligned}
\check{V}_{(p)}^{(r)}\left(\mathbb{M}^{(r)}, \gamma_{(p)}^{(r)}\right) & =\operatorname{stat}_{\check{\boldsymbol{\sigma}}_{(p)}^{(r)}}\left\{\operatorname{stat}_{\check{\boldsymbol{\epsilon}}_{(p)}^{(r)}}\left[\check{\boldsymbol{\epsilon}}_{(p)}^{(r)} \cdot \check{\boldsymbol{\sigma}}_{(p)}^{(r)}-w_{L}^{(r)}\left(\check{\boldsymbol{\epsilon}}_{(p)}^{(r)}\right)\right]-u^{(r)}\left(\check{\boldsymbol{\sigma}}_{(p)}^{(r)}\right)\right\} \\
& =\operatorname{stat}_{\check{\boldsymbol{\epsilon}}_{(p)}^{(r)}}\left\{\operatorname{stat}_{\check{\boldsymbol{\sigma}}_{(p)}^{(r)}}\left[\check{\boldsymbol{\epsilon}}_{(p)}^{(r)} \cdot \check{\boldsymbol{\sigma}}_{(p)}^{(r)}-u^{(r)}\left(\check{\boldsymbol{\sigma}}_{(p)}^{(r)}\right)\right]-w_{L}^{(r)}\left(\check{\boldsymbol{\epsilon}}_{(p)}^{(r)}\right)\right\} \\
& =\operatorname{stat}_{\check{\boldsymbol{\epsilon}}_{(p)}^{(r)}}^{(r)}\left\{w^{(r)}\left(\check{\boldsymbol{\epsilon}}_{(p)}^{(r)}\right)-w_{L}^{(r)}\left(\check{\boldsymbol{\epsilon}}_{(p)}^{(r)}\right)\right\} .
\end{aligned}
$$

In connection with this development, it should be remarked that the interchange of the stationary operations is justified by the fact that our attention is focused on a single stationary point, as described either by $\check{\boldsymbol{\sigma}}_{(p)}^{(r)}$, or by the dual variable $\check{\boldsymbol{\epsilon}}_{(p)}^{(r)}$. 
Then, making use of expression (70) in the last line in expression (71), we define the nonlinear error functions in terms of the dual variables $\mathbb{L}^{(r)}$ and $\boldsymbol{\tau}_{(p)}^{(r)}$ via the expressions

$$
\check{V}_{(p)}^{(r)}\left(\mathbb{L}^{(r)}, \boldsymbol{\tau}_{(p)}^{(r)}\right)=\operatorname{stat}_{\check{\boldsymbol{\epsilon}}_{(p)}^{(r)}}\left\{w^{(r)}\left(\check{\boldsymbol{\epsilon}}_{(p)}^{(r)}\right)-\frac{1}{2} \check{\boldsymbol{\epsilon}}_{(p)}^{(r)} \cdot \mathbb{L}^{(r)} \check{\boldsymbol{\epsilon}}_{(p)}^{(r)}-\boldsymbol{\tau}_{(r)}^{(p)} \cdot \check{\boldsymbol{\epsilon}}_{(p)}^{(r)}-\frac{1}{2} \boldsymbol{\tau}_{(p)}^{(r)} \cdot\left(\mathbb{L}^{(r)}\right)^{-1} \boldsymbol{\tau}_{(p)}^{(r)}\right\}
$$

and similarly for the functions $\hat{V}_{(p)}^{(r)}$. The slight abuse in notation in using the same symbols $\check{V}_{(p)}^{(r)}$ and $\hat{V}_{(p)}^{(r)}$ for the error functions in terms of the dual variables can be justified because these error functions have precisely the same values whether expressed in terms of the variables $\mathbb{M}^{(r)}$ and $\boldsymbol{\gamma}_{(p)}^{(r)}$, or in terms of the variables $\mathbb{L}^{(r)}$ and $\boldsymbol{\tau}_{(p)}^{(r)}$, as long as the two sets of variables are related by the duality expressions (65) - and provided that the extra quadratic terms in the eigenstresses $\boldsymbol{\tau}_{(p)}^{(r)}$ are included. In addition, paralleling definition (42), we define the corresponding compacted error functions

$$
V_{(p)}^{(r)}\left(\mathbb{L}^{(r)}, \boldsymbol{\tau}_{(p)}^{(r)}\right)=\alpha^{(r)} \check{V}_{(p)}^{(r)}\left(\mathbb{L}^{(r)}, \boldsymbol{\tau}_{(p)}^{(r)}\right)+\left(1-\alpha^{(r)}\right) \hat{V}_{(p)}^{(r)}\left(\mathbb{L}^{(r)}, \boldsymbol{\tau}_{(p)}^{(r)}\right)=V_{(p)}^{(r)}\left(\mathbb{M}^{(r)}, \boldsymbol{\gamma}_{(p)}^{(r)}\right)
$$

Then, evaluating the Legendre transform of the variational estimate $\widetilde{U}_{N}$ for $\widetilde{U}$, as given by (34), we obtain the variational estimate $\widetilde{W}_{N}$ for the macroscopic potential $\widetilde{W}$ of the nonlinear composite as

$$
\begin{aligned}
\widetilde{W}_{N}(\overline{\boldsymbol{\sigma}}) & =\operatorname{stat}_{\overline{\boldsymbol{\sigma}}}\left\{\overline{\boldsymbol{\sigma}} \cdot \overline{\boldsymbol{\epsilon}}-\operatorname{stat}_{\boldsymbol{\gamma}_{(p)}^{(s)}, \mathbb{M}^{(s)}}\left\{\widetilde{U}_{L}(\overline{\boldsymbol{\sigma}})-\sum_{r=1}^{N} c^{(r)} \sum_{p=1}^{M} \beta_{(p)}^{(r)} V_{(p)}^{(r)}\left(\mathbb{M}^{(r)}, \boldsymbol{\gamma}_{(p)}^{(r)}\right)\right\}\right\} \\
& \left.=\underset{\substack{\gamma_{(p)}^{(s)}, \mathbb{M}^{(s)} \\
\operatorname{stat}}}{\operatorname{stat}}\left\{\overline{\boldsymbol{\sigma}} \cdot \overline{\boldsymbol{\epsilon}}-\widetilde{U}_{L}(\overline{\boldsymbol{\sigma}})\right\}+\sum_{r=1}^{N} c^{(r)} \sum_{p=1}^{M} \beta_{(p)}^{(r)} V_{(p)}^{(r)}\left(\mathbb{M}^{(r)}, \boldsymbol{\gamma}_{(p)}^{(r)}\right)\right\} \\
& =\underset{\substack{\boldsymbol{\gamma}_{(p)}^{(s)}, \mathbb{M}(s) \\
\text { stat }}}{\sin }\left\{\widetilde{W}_{L}(\overline{\boldsymbol{\epsilon}})+\sum_{r=1}^{N} c^{(r)} \sum_{p=1}^{M} \beta_{(p)}^{(r)} V_{(p)}^{(r)}\left(\mathbb{M}^{(r)}, \boldsymbol{\gamma}_{(p)}^{(r)}\right)\right\},
\end{aligned}
$$

where $\widetilde{W}_{L}$ is the effective stress potential of the LCC, which was obtained via the Legendre transform of the corresponding effective strain potential $\widetilde{U}_{L}$, as given by expression (17). Finally, making use of the duality relations (65) to express the variables $\mathbb{M}^{(r)}$ and $\boldsymbol{\gamma}_{(p)}^{(r)}$ in terms of the variables $\mathbb{L}^{(r)}$ and $\boldsymbol{\tau}_{(p)}^{(r)}$, it is concluded that

$$
\widetilde{W}_{N}(\overline{\boldsymbol{\sigma}})=\operatorname{stat}_{\boldsymbol{\tau}_{(p)}^{(s)}, \mathbb{L}^{(s)}}\left\{\widetilde{W}_{L}(\overline{\boldsymbol{\epsilon}})+\sum_{r=1}^{N} c^{(r)} \sum_{p=1}^{M} \beta_{(p)}^{(r)} V_{(p)}^{(r)}\left(\mathbb{L}^{(r)}, \boldsymbol{\tau}_{(p)}^{(r)}\right)\right\},
$$


where $\widetilde{W}_{L}$ is given by (67) and the functions $V_{(p)}^{(r)}$ is given by expressions (73) in terms of $\check{V}_{(p)}^{(r)}$, as defined by expressions $(72)$, and similarly for $\hat{V}_{(p)}^{(r)}$.

It is important to emphasize that the fully stationary estimate (75) for the effective stress potential $\widetilde{W}$ of the nonlinear composite is exactly equivalent to the estimate (41) for the corresponding effective strain potential $\widetilde{U}$ (provided that the same selection is made for the weight factors $\alpha^{(r)}$ and $\beta_{(p)}^{(r)}$ in these estimates). As we have seen, this follows from the Legendre duality of the associated stress and strain potentials, $\widetilde{W}_{L}$ and $\widetilde{U}_{L}$ for the LCC, as well as the equivalence of the functions $\check{V}_{(p)}^{(r)}$ and $\hat{V}_{(p)}^{(r)}$ in these estimates. In this connection, it is interesting to remark that the stress potentials $w_{L}^{(r)}$ for the LCC in the estimate (75) for $\widetilde{W}_{N}$, as given by expressions (64), and the associated error functions in terms of the properties of the LCC, as determined by expressions of the type (72) in terms of the variables $\mathbb{L}^{(r)}$ and $\boldsymbol{\tau}_{(p)}^{(r)}$, contain 'extra' terms that are quadratic in the eigenstresses $\boldsymbol{\tau}_{(p)}^{(r)}$. It is then natural to ask whether such terms could cancel out from the expression (75) for $\widetilde{W}_{N}$. If this was the case, it would lead to a result that is more similar to the corresponding estimate (41) for $\widetilde{U}_{N}$, which does not contain such quadratic terms in the eigenstrains $\gamma_{(p)}^{(r)}$. By writing the functions $\widetilde{W}_{L}, \check{V}_{(p)}^{(r)}$ and $\hat{V}_{(p)}^{(r)}$ explicitly in the estimate $(75)$ for $\widetilde{W}_{N}$, it can be seen that the condition for these terms to cancel reduces to

$$
\left[\sum_{p=1}^{M} \beta_{(p)}^{(r)} \boldsymbol{\tau}_{(p)}^{(r)}\right] \cdot\left(\mathbb{L}^{(r)}\right)^{-1}\left[\sum_{p=1}^{M} \beta_{(p)}^{(r)} \boldsymbol{\tau}_{(p)}^{(r)}\right]=\sum_{p=1}^{M} \beta_{(p)}^{(r)} \boldsymbol{\tau}_{(p)}^{(r)} \cdot\left(\mathbb{L}^{(r)}\right)^{-1} \boldsymbol{\tau}_{(p)}^{(r)},
$$

which can obviously not be satisfied in general. However, there are some special cases where the equality is satisfied and the quadratic terms in the local potential (70) for the LCC and in the error functions (72) cancel out from the fully stationary estimate (75). In such cases, the fully stationary estimate $\widetilde{W}_{N}$ can still be written in the form (75), but now the LCC has phase stress potentials

$$
w_{L(p)}^{(r)}(\boldsymbol{\epsilon})=\frac{1}{2} \boldsymbol{\epsilon} \cdot \mathbb{L}^{(r)} \boldsymbol{\epsilon}+\boldsymbol{\tau}_{(r)}^{(p)} \cdot \boldsymbol{\epsilon}
$$

and the error functions can be written in the form

$$
\check{V}_{(p)}^{(r)}\left(\mathbb{L}^{(r)}, \boldsymbol{\tau}_{(p)}^{(r)}\right)=\operatorname{stat}_{\check{\boldsymbol{\epsilon}}_{(p)}^{(r)}}\left\{w^{(r)}\left(\check{\boldsymbol{\epsilon}}_{(p)}^{(r)}\right)-\frac{1}{2} \check{\boldsymbol{\epsilon}}_{(p)}^{(r)} \cdot \mathbb{L}^{(r)} \check{\boldsymbol{\epsilon}}_{(p)}^{(r)}-\boldsymbol{\tau}_{(r)}^{(p)} \cdot \check{\boldsymbol{\epsilon}}_{(p)}^{(r)}\right\}
$$

and similarly for $\hat{V}_{(p)}^{(r)}$. 
More generally (i.e., when the condition (76) is not satisfied), the above-described 'simplified' estimate (75) with LCC defined by (77) and error functions (78) still provides an alternative estimates for $\widetilde{W}$, as can be shown by following a procedure completely analogous to that used in the derivation of the fully stationary estimate (41) for $\widetilde{U}$. However, in

this case, the corresponding 'simplified' (dual) estimate for $\widetilde{U}$ would contain extra quadratic terms in the eigenstrains in order to ensure duality.

Therefore, in conclusion, it has been shown that the new fully stationary estimates are free of the duality gap that was present in the earlier second-order estimates (Ponte Castañeda, 1996, 2002a). However, one must be careful to keep the appropriate quadratic terms in the eigenstrains or eigenstresses to ensure the Legendre duality of the LCC potentials, as well as for the error functions. Such terms may cancel out in some special cases, but in general they do not and must be kept in order to ensure complete duality. Having made that clear, it is important to emphasize that there may be more than one fully stationary estimate, depending on the starting point-i.e., on whether the effective strain potential, or effective stress potential of the composite is used as the starting point. The important feature of these estimates, however, is that regardless of the starting point, there is a dual formulation involving precisely the same LCC. Again, this feature was not present in the earlier second order estimates of Ponte Castañeda (1996) and Ponte Castañeda (2002a). It was present in the variational bounds of Ponte Castañeda (1991), which make use of a simpler LCC not involving eigenstresses or eigenstrains, and which are not exact to second-order in the contrast.

\section{The special case of crystalline phases}

The estimate (41) for the macroscopic potential $\widetilde{U}$ is valid for nonlinear composites with general anisotropic behavior for the constituent phases, as characterized by phase potentials $u^{(r)}$. In particular, it can be used for viscoplastic single-crystal phases with constitutive response characterized by equations (6) and (7), which is of special interest in solid mechanics because most metals appear in polycrystalline form. In this section, however, we develop an alternative, simplified approach for crystalline composites and polycrystals, which exploits the special mathematical structure of these potentials, building on the earlier work of 
deBotton and Ponte Castañeda (1995) and Liu and Ponte Castañeda (2004).

With this goal in mind, we introduce an LCC with phase strain potentials given by

$$
u_{L}^{(r)}(\boldsymbol{\sigma})=\sum_{k=1}^{K} \phi_{L}^{(r)}\left(\tau_{(k)}^{(r)}\right)
$$

where it is recalled that the $\tau_{(k)}^{(r)}$ are the resolved shear stresses defined by expression (7), and the functions $\phi_{L} \underset{(k)}{(r)}$ are quadratic slip potentials defined by

$$
\phi_{L}^{(k)}(\tau)=\frac{1}{4 \mu_{(k)}^{(r)}} \tau^{2}+\gamma_{(k)}^{(r)} \tau
$$

in terms of a slip compliance $\mu_{(k)}^{(r)}$ and and a slip eigenstrain $\gamma_{(k)}^{(r)}(r=1, \ldots, N ; s=1, \ldots, K)$. It is easy to see that such potentials can be put in the form (15) by letting

$$
\mathbb{M}^{(r)}=\sum_{k=1}^{K} \frac{1}{2 \mu_{(k)}^{(r)}} \boldsymbol{\mu}_{(k)}^{(r)} \otimes \boldsymbol{\mu}_{(k)}^{(r)}, \quad \text { and } \quad \gamma^{(r)}=\sum_{k=1}^{K} \gamma_{(k)}^{(r)} \boldsymbol{\mu}_{(k)}^{(r)},
$$

where is recalled that the $\boldsymbol{\mu}_{(k)}^{(r)}(r=1, \ldots, N ; s=1, \ldots, K)$ correspond to the slip tensors that have been defined in the context of expression (7).

As in the general case, the potential $u^{(r)}$ of phase $r$ the nonlinear composite can be written in the form given by expression (20) and it is possible to follow a procedure similar to that used in obtaining expression (29). However, instead of attempting to characterize all the stationary points of the error functions $\check{V}^{(r)}$ and $\hat{V}^{(r)}$ as was done in section 3 , it is useful to carry out additional 'relaxation' steps exploiting the 'additive' form of the potentials $u^{(r)}$ and $u_{L}^{(r)}$, as given by expressions (6) and (79). Thus,

$$
\begin{aligned}
\check{V}^{(r)}\left(\mathbb{M}^{(r)}, \gamma^{(r)}\right) & =\operatorname{stat}_{\check{\boldsymbol{\sigma}}^{(r)}}\left\{\frac{1}{2} \check{\boldsymbol{\sigma}}^{(r)} \cdot \mathbb{M}^{(r)} \check{\boldsymbol{\sigma}}^{(r)}+\boldsymbol{\gamma}^{(r)} \cdot \check{\boldsymbol{\sigma}}^{(r)}-u^{(r)}\left(\check{\boldsymbol{\sigma}}^{(r)}\right)\right\} \\
& =\operatorname{stat}_{\check{\boldsymbol{\sigma}}^{(r)}} \sum_{k=1}^{K}\left\{\frac{1}{4 \mu_{(k)}^{(r)}}\left(\check{\boldsymbol{\sigma}}^{(r)} \cdot \mu_{(k)}^{(r)}\right)^{2}+\gamma_{(k)}^{(r)}\left(\check{\boldsymbol{\sigma}}^{(r)} \cdot \mu_{(k)}^{(r)}\right)-\phi_{(k)}^{(r)}\left(\check{\boldsymbol{\sigma}}^{(r)} \cdot \mu_{(k)}^{(r)}\right)\right\} \\
& \approx \sum_{k=1}^{K} \operatorname{stat}_{\check{\tau}_{(k)}^{(r)}}\left\{\frac{1}{4 \mu_{(k)}^{(r)}}\left(\check{\tau}_{(k)}^{(r)}\right)^{2}+\gamma_{(k)}^{(r)} \check{\tau}_{(k)}^{(r)}-\phi_{(k)}^{(r)}\left(\check{\tau}_{(k)}^{(r)}\right)\right\} \\
& =\sum_{k=1}^{K} \check{V}_{(k)}^{(r)}\left(\mu_{(k)}^{(r)}, \gamma_{(k)}^{(r)}\right),
\end{aligned}
$$

where

$$
\check{V}_{(k)}^{(r)}\left(\mu_{(k)}^{(r)}, \gamma_{(k)}^{(r)}\right)=\operatorname{stat}_{\check{\tau}_{(k)}^{(r)}}\left\{\frac{1}{4 \mu_{(k)}^{(r)}}\left(\check{\tau}_{(k)}^{(r)}\right)^{2}+\gamma_{(k)}^{(r)} \check{\tau}_{(k)}^{(r)}-\phi_{(k)}^{(r)}\left(\check{\tau}_{(k)}^{(r)}\right)\right\} .
$$


It should be emphasized in connection with expression (82) that the replacement of the 'difficult' optimization over the tensors $\check{\boldsymbol{\sigma}}^{(r)}$ of a sum of functions by the sum of 'simpler' optimization problems over the scalar variables $\check{\tau}_{(k)}^{(r)}$ will, in general, lead to different results, and hence the use of the approximate equal sign in the expression. However, as shown recently by Ponte Castañeda (2015), it is still possible to show that the local potential $u^{(r)}$ of the nonlinear composite can be recovered exactly by an expression analogous to (29), but where the optimization over the tensors $\mathbb{M}^{(r)}$ and $\gamma^{(r)}$ is replaced by the (larger) set of scalar variables $\mu_{(k)}^{(r)}$ and $\gamma_{(k)}^{(r)}$. Thus, making use of an expression completely analogous to expression (82) for the function $\hat{V}^{(r)}$ in terms of similarly defined (cf. (83)) functions $\hat{V}_{(k)}^{(r)}$, and of the definition

$$
V_{(k)}^{(r)}\left(\mu_{(k)}^{(r)}, \gamma_{(k)}^{(r)}\right)=\alpha^{(r)} \check{V}_{(k)}^{(r)}\left(\mu_{(k)}^{(r)}, \gamma_{(k)}^{(r)}\right)+\left(1-\alpha^{(r)}\right) \hat{V}_{(k)}^{(r)}\left(\mu_{(k)}^{(r)}, \gamma_{(k)}^{(r)}\right)
$$

which is similar to expression (30), it was shown by Ponte Castañeda (2015) that

$$
u^{(r)}(\boldsymbol{\sigma})=\operatorname{stat}_{\substack{(r) \\ \mu_{(n)}, \gamma_{(n)}^{(r)}}}\left\{u_{L}^{(r)}(\boldsymbol{\sigma})-\sum_{k=1}^{K^{(r)}} V_{(k)}^{(r)}\left(\mu_{(k)}^{(r)}, \gamma_{(k)}^{(r)}\right)\right\} .
$$

Then, by making use of the definition (11) for the macroscopic potential $\widetilde{U}$ of the nonlinear composite, it was shown by Ponte Castañeda (2015) that the macroscopic potential of the nonlinear composite could be corresponding estimated as

$$
\widetilde{U}(\overline{\boldsymbol{\sigma}}) \approx \widetilde{U}_{N}(\overline{\boldsymbol{\sigma}}) \operatorname{stat}_{\substack{(s) \\ \mu_{(n)}, \gamma_{(n)}^{(s)}}}\left\{\widetilde{U}_{L}(\overline{\boldsymbol{\sigma}})-\sum_{r=1}^{N} c^{(r)} \sum_{k=1}^{K^{(r)}} V_{(k)}^{(r)}\left(\mu_{(k)}^{(r)}, \gamma_{(k)}^{(r)}\right)\right\}
$$

where $\widetilde{U}_{L}$ now corresponds to the effective potential for the LCC defined by expression (17) with phase potentials defined by $(20)$ with $\mathbb{M}^{(r)}$ and $\gamma^{(r)}$ defined by expressions (81) in terms of $\mu_{(k)}^{(r)}$ and $\gamma_{(k)}^{(r)}$. Thus, expression (86) provides an alternative, generally different, but easierto-compute estimate for the effective potential $\widetilde{U}$ of nonlinear composite with single-crystal viscoplastic behavior to expression (41) for nonlinear composites with general anisotropic behavior for the phases.

\section{Application to 2-D porous, isotropic, power-law material}

In this section, the general results of section 3 are specialized for porous materials consisting of aligned cylindrical voids of circular cross section that are distributed randomly (in 
the transverse plane) in an incompressible, isotropic matrix with potential (5), in such a way that the overall symmetry of the composite is transversely isotropic and the volume fraction of the voids, or porosity is $f$. For simplicity, only plane strain loading transverse to the cylindrical voids will be considered here, so that the problem becomes two-dimensional. In addition, again for simplicity, the loading will be taken to be purely deviatoric, so that the hydrostatic component of the macroscopic stress will be assumed to vanish. Furthermore, a power law will be assumed for the matrix potential, so that the microscopic and macroscopic potentials of the composite can be written in the form (e.g., Ponte Castañeda and Suquet, 1998)

$$
u^{(1)}(\boldsymbol{\sigma})=\phi^{(1)}\left(\sigma_{e}\right)=\frac{\sigma_{0}}{n+1}\left(\frac{\sigma_{e}}{\sigma_{0}}\right)^{n+1}, \quad \text { and } \tilde{U}(\overline{\boldsymbol{\sigma}})=\frac{\tilde{\sigma}_{0}}{n+1}\left(\frac{\bar{\sigma}_{e}}{\tilde{\sigma}_{0}}\right)^{n+1},
$$

where $n(1 \leq n<\infty)$ is the nonlinearity exponent and $\sigma_{0}$ and $\tilde{\sigma}_{0}$ are the matrix and macroscopic flow stresses, respectively. Thus, the homogenized response of this two-dimensional porous power-law material is determined by the ratio $\tilde{\sigma}_{0} / \sigma_{0}$ as a function of the nonlinearity $n$, the porosity $f$ and additional microstructural information, such isotropy of the two-point correlations.

For the purpose of carrying out the implementation of the new homogenization method for this special case, the LCC is taken to be characterized by a matrix compliance tensor of the form

$$
\mathbb{M}^{(1)}=\frac{1}{2 \lambda} \mathbb{E}+\frac{1}{2 \mu} \mathbb{F}
$$

where

$$
\mathbb{E}=\frac{3}{2 \bar{\sigma}_{e}^{2}} \overline{\boldsymbol{s}} \otimes \overline{\boldsymbol{s}}, \quad \text { and } \quad \mathbb{F}=\mathbb{K}-\mathbb{E}
$$

are projection operators, such that $\mathbb{E} \mathbb{E}=\mathbb{E}, \mathbb{F} \mathbb{F}=\mathbb{F}, \quad \mathbb{E} \mathbb{F}=\mathbb{F} \mathbb{E}=\mathbb{O}, \quad \mathbb{J}+\mathbb{E}+\mathbb{F}=\mathbb{I}$ (Ponte Castañeda, 1996). In the above relations, $\mathbb{K}$ and $\mathbb{J}$ are the standard fourth-order, isotropic, shear and hydrostatic projection tensors, respectively. On the other hand, the matrix eigenstrain (38) is taken to be of the form

$$
\gamma^{(1)}=\frac{1}{2}\left(\gamma_{(1)}^{(1)}+\gamma_{(2)}^{(1)}\right)=\frac{1}{2}\left(\gamma^{I}+\gamma^{I I}\right)
$$

where the $\beta_{(p)}^{(1)}(p=1, \ldots, 2)$ have been taken to be equal to one half, and simplified notation has been introduced for the corresponding $\gamma_{(p)}^{(1)}$. This is because, while the individual eigen- 
strains $\gamma_{(k)}^{(1)}$ will be found to have components that are not aligned with the macroscopic stress $\overline{\boldsymbol{\sigma}}$, the overall eigenstrain $\boldsymbol{\gamma}^{(1)}$ will need to be aligned with $\overline{\boldsymbol{\sigma}}$.

Now, the porous phase is characterized by a null potential, and the macroscopic response and field statistics of the porous LCC may be determined in terms of the porosity $f$ and additional microstructural information for the distribution of the voids. In this work, we assume that the circular voids are distributed isotropically in the transverse plane and make use of the Hashin-Shtrikman-type estimates of Ponte Castañeda and Willis (1995); in particular, the matrix average of the stress and the covariance of the stress field fluctuations are given by (Ponte Castañeda, 2002b)

$$
\overline{\boldsymbol{\sigma}}^{(1)}=\frac{1}{1-f} \overline{\boldsymbol{\sigma}}, \quad \mathbb{C}_{\boldsymbol{\sigma}}^{(1)}=\frac{1}{2} \frac{f}{(1-f)^{2}} \frac{1}{\sqrt{\lambda \mu}}\left(\mathbb{M}^{(1)}\right)^{-1} .
$$

Next, in order to spell out the stationarity conditions (43) to (46), it is useful to introduce the consistent notation $\check{\boldsymbol{\sigma}}^{I}$ and $\check{\boldsymbol{\sigma}}^{I I}$ for $\check{\boldsymbol{\sigma}}_{(1)}^{(1)}$ and $\check{\boldsymbol{\sigma}}_{(1)}^{(2)}$, respectively, in the definition of (40) for the function $\check{V}_{(p)}^{(r)}$, and similarly for $\hat{\boldsymbol{\sigma}}^{I}$ and $\hat{\boldsymbol{\sigma}}^{I I}$ in the definition of $\hat{V}_{(p)}^{(r)}$. Now, to satisfy the overall symmetry requirements of the problem, it is necessary that the tensors $\check{\boldsymbol{\sigma}}^{I}, \hat{\boldsymbol{\sigma}}^{I}$ and $\check{\boldsymbol{\sigma}}^{I I}, \hat{\boldsymbol{\sigma}}^{I I}$ be arranged symmetrically about the loading direction defined by $\overline{\boldsymbol{\sigma}}$. Referring to Fig. 1(b), when $\boldsymbol{\gamma}^{(1)}$ does not vanish, the picture in this figure needs to be rotated clockwise, or counterclockwise, depending on the direction of $\gamma^{(1)}$. As a consequence, the two saddle points corresponding to the locations of the variables $\check{\boldsymbol{\sigma}}^{I}$ and $\hat{\boldsymbol{\sigma}}^{I}$, and the two other saddle points corresponding to the variables $\check{\boldsymbol{\sigma}}^{I I}$ and $\hat{\boldsymbol{\sigma}}^{I I}$ need to be arranged symmetrically about the loading direction (i.e., rotated clockwise and counterclockwise, respectively, but by equal amounts). For these reason, it is useful to define the 'parallel' and 'perpendicular' components (relative to the loading direction, as defined by $\overline{\boldsymbol{\sigma}}$ ) of the tensors $\check{\boldsymbol{\sigma}}^{I}, \hat{\boldsymbol{\sigma}}^{I}, \boldsymbol{\gamma}^{I}$, etc. For example, $\check{\sigma}_{\|}^{I}$ and $\check{\sigma}_{\perp}^{I}$ are the parallel and perpendicular components of $\check{\boldsymbol{\sigma}}^{I}$, such that $\check{\sigma}_{e}^{I}=\sqrt{\left(\check{\sigma}_{\|}^{I}\right)^{2}+\left(\check{\sigma}_{\perp}^{I}\right)^{2}}$-and similarly for $\hat{\sigma}_{\|}^{I}, \hat{\sigma}_{\perp}^{I}$, and $\gamma_{\|}^{I}, \gamma_{\perp}^{I}$, etc. It can then be shown that the optimality conditions (43) reduce to

$$
\gamma_{\|}^{I}=\gamma_{\|}^{I I}=\gamma_{\|}=\left[\left(\frac{\hat{\sigma}_{e}}{\sigma_{0}}\right)^{n-1}-\frac{\sigma_{0}}{3 \lambda}\right] \frac{\hat{\sigma}_{\|}}{\sigma_{0}}=\left[\left(\frac{\check{\sigma}_{e}}{\sigma_{0}}\right)^{n-1}-\frac{\sigma_{0}}{3 \lambda}\right] \frac{\check{\sigma}_{\|}}{\sigma_{0}},
$$

and

$$
\gamma_{\perp}^{I}=-\gamma_{\perp}^{I I}=\gamma_{\perp}=\left[\left(\frac{\hat{\sigma}_{e}}{\sigma_{0}}\right)^{n-1}-\frac{\sigma_{0}}{3 \mu}\right] \frac{\hat{\sigma}_{\perp}}{\sigma_{0}}=\left[\left(\frac{\check{\sigma}_{e}}{\sigma_{0}}\right)^{n-1}-\frac{\sigma_{0}}{3 \mu}\right] \frac{\check{\sigma}_{\perp}}{\sigma_{0}},
$$


where $\check{\sigma}_{\|}=\left(\frac{3}{2} \check{\boldsymbol{\sigma}} \cdot \mathbb{E} \check{\boldsymbol{\sigma}}\right)^{\frac{1}{2}}$ and $\check{\sigma}_{\perp}=\left(\frac{3}{2} \check{\boldsymbol{\sigma}} \cdot \mathbb{F} \check{\boldsymbol{\sigma}}\right)^{\frac{1}{2}}$, and where use has been made of the symmetry of the problem in setting $\check{\sigma}_{\|}^{I}=\check{\sigma}_{\|}^{I I}=\check{\sigma}_{\|}, \check{\sigma}_{\perp}^{I}=-\check{\sigma}_{\perp}^{I I}=\check{\sigma}_{\perp}, \hat{\sigma}_{\|}^{I}=\hat{\sigma}_{\|}^{I I}=\hat{\sigma}_{\|}$and $\hat{\sigma}_{\perp}^{I}=-\hat{\sigma}_{\perp}^{I I}=$ $\hat{\sigma}_{\perp}$.

As already noted, the variational estimate (41) is fully stationary for any choice of $\alpha^{(1)}$. In this example, we will make use of the choice $\alpha^{(1)}=1 / 2$, which as discussed earlier leads to pairs of stationary points $\check{\boldsymbol{\sigma}}^{I}$ and $\hat{\boldsymbol{\sigma}}^{I}$, and $\check{\boldsymbol{\sigma}}^{I I}$ and $\hat{\boldsymbol{\sigma}}^{I I}$, respectively, that are symmetrically distributed about the phase average $\overline{\boldsymbol{\sigma}}^{(1)}$. Then, the stationarity conditions (47) over the variables $\boldsymbol{\gamma}^{I}$ and $\boldsymbol{\gamma}^{I I}$ lead to corresponding conditions for $\check{\boldsymbol{\sigma}}^{I}$ and $\hat{\boldsymbol{\sigma}}^{I}$, and $\check{\boldsymbol{\sigma}}^{I I}$ and $\hat{\boldsymbol{\sigma}}^{I I}$, which on account of the above-mentioned symmetry conditions for the parallel and perpendicular components of these variables (and choice for $\alpha^{(1)}$ ) can be shown to reduce to

$$
\frac{1}{2}\left(\check{\sigma}_{\|}+\hat{\sigma}_{\|}\right)=\bar{\sigma}_{e}^{(1)}=\frac{1}{1-f} \bar{\sigma}_{e}, \quad \text { and } \quad \check{\sigma}_{\perp}+\hat{\sigma}_{\perp}=0
$$

On the other hand, the conditions (48) for the field fluctuations, again after simplification to account for the symmetry conditions, can be shown to reduce to

$$
\begin{aligned}
\left(\hat{\sigma}_{\|}-\bar{\sigma}_{e}^{(1)}\right)^{2} & =\left(\bar{\sigma}_{e}^{(1)}-\check{\sigma}_{\|}\right)^{2}=\frac{3}{2} \mathbb{C}_{\boldsymbol{\sigma}}^{(r)} \cdot \mathbb{E}=\frac{f \sqrt{k}}{2(1-f)^{2}} \bar{\sigma}_{e}^{2} \\
\left(\hat{\sigma}_{\perp}\right)^{2} & =\left(-\check{\sigma}_{\perp}\right)^{2}=\frac{3}{2} \mathbb{C}_{\boldsymbol{\sigma}}^{(r)} \cdot \mathbb{F}=\frac{f}{2(1-f)^{2} \sqrt{k}} \bar{\sigma}_{e}^{2}
\end{aligned}
$$

where we have made use of expressions (91), and where $k=\lambda / \mu$ is the anisotropy ratio of the LCC matrix material.

Now, the equations (94) and (96) can be solved for $\hat{\sigma}_{\|}, \hat{\sigma}_{\perp}, \check{\sigma}_{\|}$and $\check{\sigma}_{\perp}$ to obtain the results

$$
\begin{aligned}
& \hat{\sigma}_{\|}=\left(1+\sqrt{\frac{f \sqrt{k}}{2}}\right)\left(\frac{\bar{\sigma}_{e}}{1-f}\right), \hat{\sigma}_{\perp}=\sqrt{\frac{f}{2 \sqrt{k}}}\left(\frac{\bar{\sigma}_{e}}{1-f}\right), \\
& \check{\sigma}_{\|}=\left(1-\sqrt{\frac{f \sqrt{k}}{2}}\right)\left(\frac{\bar{\sigma}_{e}}{1-f}\right), \check{\sigma}_{\perp}=-\sqrt{\frac{f}{2 \sqrt{k}}}\left(\frac{\bar{\sigma}_{e}}{1-f}\right),
\end{aligned}
$$

where the symmetry of the variables $\hat{\sigma}$ and $\check{\sigma}$ under the change of sign is noted.

Finally, making use of the expression (49), we arrive at the following result for the effective 
flow stress of the porous, power-law material

$$
\left[\frac{(1-f) \sigma_{0}}{\tilde{\sigma}_{0}}\right]^{n}=\frac{1}{2} \operatorname{stat}\left\{\left[\left(1-\sqrt{\frac{f \sqrt{k}}{2}}\right)^{2}+\frac{f}{2 \sqrt{k}}\right]^{\frac{n+1}{2}}+\left[\left(1+\sqrt{\frac{f \sqrt{k}}{2}}\right)^{2}+\frac{f}{2 \sqrt{k}}\right]^{\frac{n+1}{2}}\right\},
$$

where the stationarity condition provides an equation for the anisotropy ratio $k$ of the LCC as a function of $f$ and $n$, which can be written in the form

$$
\left[1-\sqrt{\frac{f}{2}}\left(\frac{1}{k^{3 / 4}}-k^{1 / 4}\right)\right]\left[\left(1+\sqrt{\frac{f \sqrt{k}}{2}}\right)^{2}+\frac{f}{2 \sqrt{k}}\right]^{\frac{n-1}{2}}=\left[1+\sqrt{\frac{f}{2}}\left(\frac{1}{k^{3 / 4}}-k^{1 / 4}\right)\right]\left[\left(1-\sqrt{\frac{f \sqrt{k}}{2}}\right)^{2}+\frac{f}{2 \sqrt{k}}\right]^{\frac{n-1}{2}} .
$$

Consistent with the general theoretical results developed in the previous sections, it is noted that the estimate (98) for the effective response of the porous power-law material can also be generated directly from the macroscopic stress-strain relation (50) for the LCC, as well as from the dual version (75) of the result. However, the algebraic derivations are a bit involved and will not be included here for brevity. In addition, explicit expressions can be easily derived for the field statistics of the porous power-law material making use of the results (19) for the phase average and second moments of the stress field (and corresponding results for the strain field). The result for the average stress in the matrix has already been given in expression $(91)_{1}$, while results for the field fluctuations are most easily obtained from expressions $(91)_{2}$ and (88) in terms of $f$ and $k$ (as characterized by expression (99) above). Analogous expressions from the dual formulation can be used to generate expressions for the phase averages and fluctuations of the strain field. For brevity, only the result for the average of the strain over the vacuous phase is given explicitly here as

$$
\frac{\bar{\epsilon}_{e}^{(2)}}{\bar{\epsilon}_{e}}=\frac{2(1+\sqrt{k})+f(1-k)}{2 \sqrt{k}+f(3-k)},
$$

where, again, $k$ is obtained from expression (99).

It can be easily verified that, in the limit of linear elastic behavior $(n=1)$, the result (98) reduces to the well known Hashin-Shtrikman (HS) upper bound, while the expression (100) for the average strain over the voids reduces to $\bar{\epsilon}_{e}^{(2)} / \bar{\epsilon}_{e}=2 /(1+f)$. On the other hand, in the ideally plastic limit $(m \rightarrow 0)$, it can be shown that the expression (98) reduces to

$$
\frac{\tilde{\sigma}_{0}}{\sigma_{0}}=\frac{(1-f)(1-k)}{\sqrt{1+k}}, \quad \text { where } \quad \frac{k^{\frac{3}{4}}}{1-k}=\sqrt{\frac{f}{2}} .
$$


Interestingly, this particular result is identical to the corresponding result of Ponte Castañeda (2002b) for the earlier version of the second-order estimates (for ideally plastic porous materials). It is recalled that the equation for $k$ is essentially a quartic equation, and can be solved analytically for $k(0<k<1)$ as a function of $f$. It is also noted that, in the limit as $f \rightarrow 0, \tilde{\sigma}_{0} / \sigma_{0} \sim 1-1.5(f / 2)^{2 / 3}$, while $\bar{\epsilon}_{e}^{(2)} / \bar{\epsilon}_{e} \sim 1+(f / 2)^{-1 / 3}$, which implies that the dilute limit is singular for the porous ideally plastic material, as will be discussed further below.

Figure 2 shows the results of the fully optimized (i.e., fully stationary) second-order (FOSO) estimates for the effective flow stress $\tilde{\sigma}_{0}$, normalized by the flow stress $\sigma_{0}$ of the matrix, as well as the average strain of the void phase $\bar{\epsilon}_{e}^{(2)}$, normalized by the macroscopic strain $\bar{\epsilon}_{e}$, for porous, power-law, isotropic materials, as functions of the nonlinearity exponent $m=1 / n$ and the porosity $f$. The new results are compared with the Taylor and 'variational' HS bounds, as well as two different earlier versions of the 'second-order' estimates. The Taylor bound is obtained by making use of a uniform strain rate $(\boldsymbol{\epsilon}=\overline{\boldsymbol{\epsilon}})$ in the expression for the effective potential $\widetilde{W}$, and incorporates only information about the porosity $f$. The variational HS upper bounds are obtained by means of the variational linear comparison method of Ponte Castañeda (1991), making use of the Hashin-Shtrikman-Willis bounds (Hashin and Shtrikman, 1963; Willis, 1977) for the appropriate LCC. The tangent secondorder (TSO) estimates were proposed by Ponte Castañeda (1996) and were obtained by the linear comparison variational approach using an estimate of the Willis type (Ponte Castañeda and Willis, 1995) for the LCC, whose matrix eigenstrain was obtained by an optimization procedure, but whose modulus was chosen to be the tangent modulus. Because of the non-optimality, this method yields different predictions depending on whether the potential energy $(\mathrm{W})$ or complementary energy (U) are used as starting points. On the other hand, the partially optimized second-order (PO-SO) estimates were proposed by Ponte Castañeda (2002b) and obtained by means of the 'second-order' method of Ponte Castañeda (2002a) using the Willis estimates for the LCC, whose matrix moduli were chosen optimally, but whose matrix eigenstrain was selected in an ad hoc fashion. Depending on the starting point, there are two different version of these PO-SO estimates ( $\mathrm{U}$ and $\mathrm{W}$ ), but the LCC is actually the same for both, and leads to a third LCC estimate (which is sometimes labelled 'affine' in the literature). 


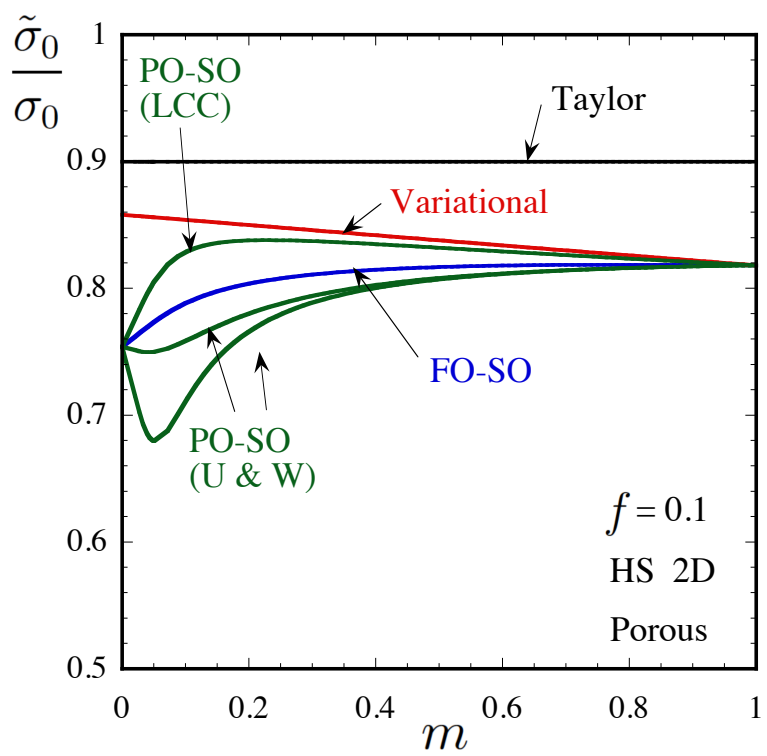

(a)

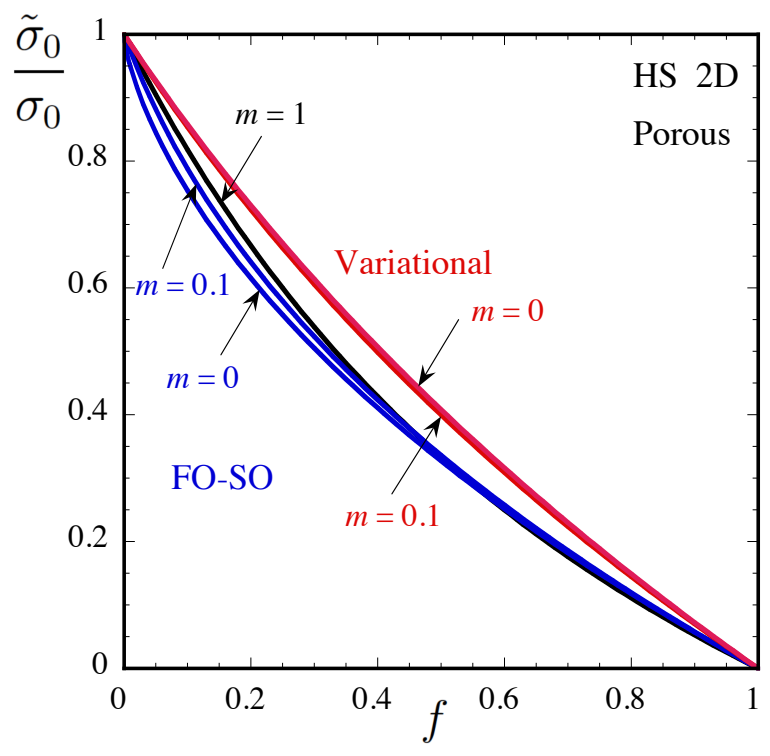

(c)

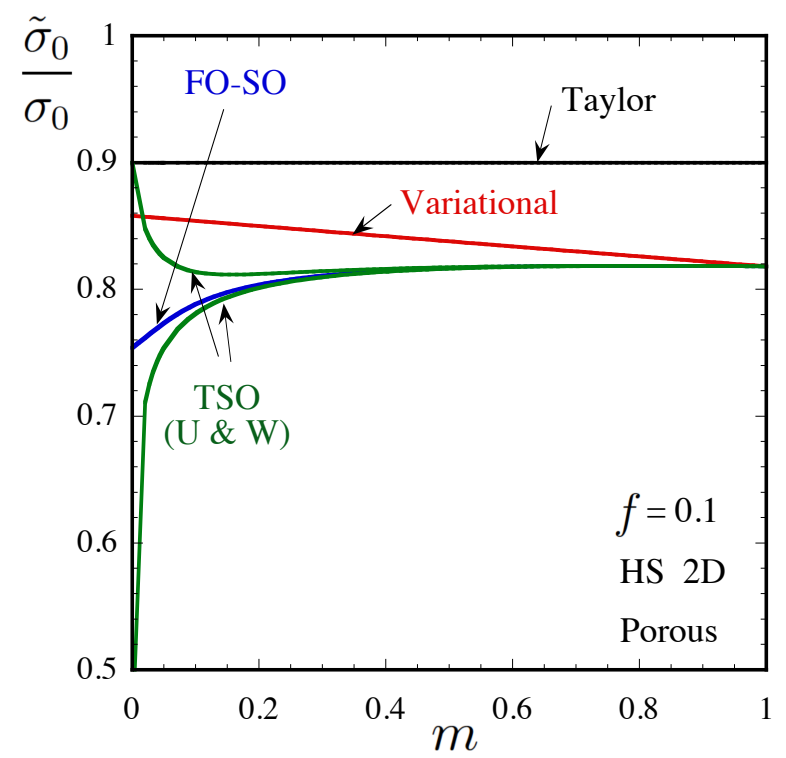

(b)

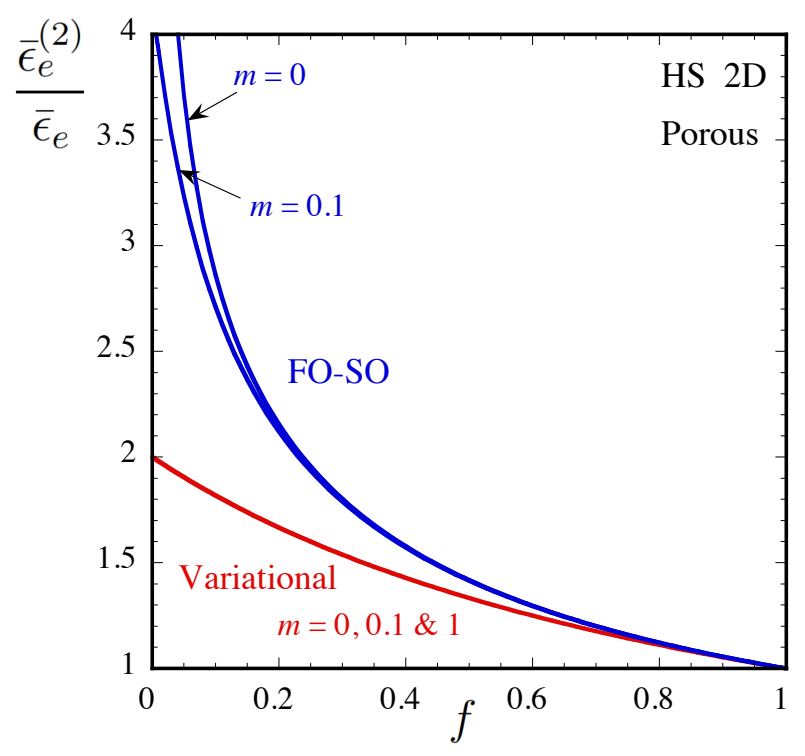

(d)

Figure 2: Estimates of the Hashin-Shtrikman (HS) type obtained by the fully optimized second-order method (FO-SO) for the normalized effective flow stress $\tilde{\sigma}_{0}$ and average strain over the pores $\bar{\epsilon}_{e}^{(2)}$ for 2-D, porous, power-law materials. (a) Results for $\tilde{\sigma}_{0} / \sigma_{0}$, as a function of the nonlinearity exponent $m=1 / n$, are compared with the Taylor and 'variational' HS upper bounds, as well as with the earlier version (PO-SO) of the secondorder estimates ( $U, W$ and LCC versions) of Ponte Castañeda (2002b). (b) Similar results but compared with the tangent second-order (TSO) estimates (U and W) of Ponte Castañeda (1996). (c) Results for $\tilde{\sigma}_{0} / \sigma_{0}$, as a function of the porosity $f$, are compared with the variational HS upper bounds for 3 different values of $m$ $(0,0.1$ and 1$)$. (d) Results for $\bar{\epsilon}_{e}^{(2)} / \bar{\epsilon}_{e}$, as a function of the porosity $f$, are compared with the variational HS estimates for 3 different values of $m(0,0.1$ and 1$)$. 
Thus, Fig. 2(a) shows a comparison of the new FO-SO estimate with the Taylor and variational HS bound, as well as the three different versions of the PO-SO estimate (U, W and LCC), as functions of the nonlinearity $m$, for a fixed value of the porosity $(f=0.1)$. It can be seen that the FO-SO estimate satisfies the bounds and is roughly in between the various predictions of the PO-SO. In fact, it agrees with the variational bound and PO-SO estimate in the linear limit $(m=1)$, as expected, but more interestingly, it also agrees with the various versions of the PO-SO estimate in the ideally plastic limit $(m=0)$, where the duality gap in the earlier PO-SO estimates vanishes. Nevertheless, the new FO-SO estimate is seen to provide significant improvements over the earlier PO-SO version for intermediate values of the nonlinearity $m$. In particular, for values of $m$ between 0.05 and 0.1 , the differences can exceed $10 \%$. In addition the dependence of the new estimate on $m$ is the opposite of the 'variational' bound - it decreases monotonically with increasing values of the nonlinearity $n$ (decreasing $m$ ), instead of increasing with $n$. This is more consistent with the result of numerical simulations by Idiart et al. (2006) using the FFT method, albeit for weaker particles and not precisely voids. It is also different from the $\mathrm{W}$ and $\mathrm{U}$ versions of the earlier PO-SO estimates which exhibit non-monotonic behavior for $m$ between 0 and 0.05 . On the other hand, Fig. 2(b) shows the corresponding comparison of the new FO-SO estimate with the two different versions of the earlier TSO estimate ( $U$ and $W$ ). It is interesting to remark that the new FO-SO estimate is in excellent agreement with the TSO estimate for small values of the nonlinearity $(m \geq 0.4)$, where the two different versions of the TSO estimate are in perfect agreement, and it is roughly in between the two for smaller values of $m$. Figure 2(c) shows a comparison of the new FO-SO estimate with the variational HS bound as a function of the porosity $f$ for several fixed values of the nonlinearity $(m=0,0.1$ and 1). As already mentioned, these two estimates agree in the linear limit $(m=1)$, but exhibit different trends as $m \rightarrow 0$. In particular, for low porosities, the slope of the curves can be seen to increase dramatically for the new FO-SO estimate as $m \rightarrow 0$, while the corresponding slope for the variational bound actually decreases slightly. This observation is consistent with earlier observations in connection with the expression $(101)_{1}$ for ideally plastic, porous composites, which was shown to behave like $\tilde{\sigma}_{0} / \sigma_{0} \sim 1-1.5(f / 2)^{2 / 3}$ in the dilute limit. Interestingly, this singular dependence $(f / 2)^{2 / 3}$ is in precise agreement 
with recent estimates by Willot (2015) based on the length of geodesics spanning random (Boolean) sets of circular holes. Finally, Fig. 2(d) presents corresponding results for the normalized average strain in the pores $\bar{\epsilon}_{e}^{(2)} / \bar{\epsilon}_{e}$, as a function of the porosity $f$, for 3 values of the nonlinearity ( $m=0,0.1$ and 1 ). As can be seen from this plot, the new FO-SO estimate for the pore strain - unlike the corresponding variational HS estimate - is very sensitive to the nonlinearity $m$ for the smaller porosities $f$, and can be seen to increase dramatically with decreasing values of $f$ for values of $m$ approaching 0 . This is consistent with earlier remarks concerning the ideally plastic limit of expression (100), which was found to behave like $\bar{\epsilon}_{e}^{(2)} / \bar{\epsilon}_{e} \sim 1+(f / 2)^{-1 / 3}$ as $f \rightarrow 0$. Physically, this is consistent with the 'geodesics' picture in the work of Willot (2015), since the strain is concentrated on these minimum energy paths linking up the voids, while the rest of the matrix material behaves like rigid blocks (see also Idiart et al., 2006). More generally, these observations also highlight the importance of developing more refined homogenization estimates, such as the one developed in this work. While the improvements relative to earlier (coarser) homogenization methods, such as the variational method (Ponte Castañeda, 1991), may be relatively modest for the macroscopic response, the corresponding improvements relative to earlier methods can be quite significant for the first and higher moments of the fields over the phases. Due to lack of space, this point will not be pursued further here, but will be taken up in future work.

However, it is important to note that the above-described solution is not the only one possible. First, the choice of stationary stresses in the shifted potentials defining the "error functions" (42) is not unique. Indeed, we have been able to construct an alternative "fully stationary" estimate making use of only 3 stationary stresses in the error functions, instead of the 4 used in the derivation of the estimate (98). This alternative estimate involves only one $\gamma^{(1)}$, which is "aligned' with the applied stress $\overline{\boldsymbol{\sigma}}$, and makes use of the two saddle points and the local minimum in Fig. 1(b). While it can also be shown to recover the earlier second-order estimate of Ponte Castañeda (2002b) in the ideally plastic limit $(n \rightarrow \infty)$ for an appropriate choice of $\alpha^{(1)}$, it was found to give slightly smaller results for intermediate values of $n$. This could be due to the fact that the use of a local minimum (in the definition of the error functions) may introduce larger errors that the corresponding use of the saddle points. In addition, it should be emphasized that, while the choice of the weights $\beta_{(p)}^{(1)}$ in the 
estimate (98) was completely determined by the symmetries required for consistency with overall isotropy, the corresponding selection of the weight $\alpha^{(1)}$ is less clear. Here, we have made the most symmetric choice $\alpha^{(1)}=1 / 2$, which, in some sense, could be expected to lead to smaller overall errors than less symmetric choices, although it is not ruled out that there may be a better choice. But, if there is a better criterion for the selection of $\alpha^{(1)}$, it does not seem to be consistent with stationarity with respect to this variable (which, in any event, is not required for the nice properties described in section 3.4) to hold.

\section{Concluding remarks}

In this work a variational method has been advanced for estimating the macroscopic properties of nonlinear composites and polycrystals with viscoplastic phases in terms of the corresponding properties of suitably designed linear comparison composites (LCC). The phases of the LCC are characterized by general quadratic potentials in the stress or strain (including linear terms), whose properties in turn play the role of trial fields in a variational statement for the stress or strain potential of the viscoplastic composites. When fully optimized, these moduli (or compliance) tensors and eigenstresses (or eigenstrains) are found to be characterized by a certain 'generalized secant' linearization of the potentials of the nonlinear phases, and to depend on the first and second moments of the strains (or stresses) in the LCC. The resulting 'fully stationary' estimates are exact to second order in the heterogeneity contrast and are consistent with known bounds. In addition, they exhibit several desirable and useful properties that were missing in earlier estimates of the second-order type (Ponte Castañeda, 1996, 2002a), due to the lack of 'full stationarity' with respect to the properties of the phases of the LCC (in the earlier estimates). These properties are similar to those that were already available in the variational bounds of Ponte Castañeda (1991), including: $(i)$ the macroscopic response and field statistics of the nonlinear composite can be obtained directly from the corresponding macroscopic response and field statistics in the LCC, thus greatly simplifying the computation of these quantities, and ( $i i)$ the lack of a 'duality gap,' leading to more accurate predictions. Setting aside the merits of the new method, it should be noted that there are still some open questions that will require further investigation in future works. In fact, as already alluded to at the end of the previous section, 
the choice of stationary stresses in the shifted potentials defining the "error" functions (42) is not unique, and even for a given choice of such stationary points, the "symmetry" criterion described at the end of section 3.3 for selecting the values of the weights $\alpha^{(r)}$ in the fully stationary estimate (41) is not entirely satisfying - and other criteria could be envisaged. For example, the weights $\alpha^{(r)}$ could be determined by considering special limiting cases with known solutions, or by appropriate comparisons with the results numerical simulations.

By way of an illustrative example, the new method was applied to the simple (twodimensional) case of a porous, isotropic, power-law material, using estimates of the HashinShtrikman type for the appropriate LCC, and the results were compared with known bounds and earlier estimates. It was found that the new fully stationary estimates satisfy the "variational' bounds, and are in agreement with the earlier 'tangent second-order' estimates of Ponte Castañeda (1996) for weak nonlinearities, as well as with the non-optimized 'generalized secant' estimates of Ponte Castañeda (2002b) in the (strongly nonlinear) ideally plastic limit. In addition, the results for porous ideally plastic materials exhibit a non-analytic $\left(f^{2 / 3}\right)$ dependence on the porosity $f$ in the dilute limit - in agreement with recent results by Willot (2015) based on the lengths of 'geodesics' spanning random sets of (two-dimensional) voids in an ideally plastic matrix. Finally, the corresponding predictions for the average strain in the vacuous phase of these ideally plastic materials is also non-analytic, exhibiting a singular $\left(f^{-1 / 3}\right)$ dependence on the porosity in the dilute limit.

One distinct advantage of the new method is that it can be used in combination with other types of estimates - such as the self-consistent approximation-for the LCC. Indeed, the application of the method will be investigated for such other types of estimates, as well as for other types of microstructures, such as those present in porous crystals (Mbiakop et al., 2015) and polycrystalline aggregates (Liu and Ponte Castañeda, 2004), in future work. In addition, comparisons of the new estimates should also be carried out with other types of estimates, such as the results of deBotton and Hariton (2002) and Idiart (2008) for infinite-rank laminates, as well as with the results of numerical simulations (e.g., Moulinec and Suquet, 1998; Fritzen et al., 2015). Finally, it should emphasized that, although the new homogenization method developed in this work was presented in the specific context of viscoplastic composites, the general ideas of the method are expected to be useful for a large 
number of applications where nonlinear homogenized properties can be given appropriate variational formulations, such as soft elastic composites (Lopez-Pamies and Ponte Castañeda, 2004; Avazmohammadi and Ponte Castañeda, 2014) and elasto-plastic composites (Lahellec and Suquet, 2007a,b).

\section{Acknowledgement}

This work was begun with the support of the Research Award from a Alexander von Humboldt Foundation at the University of Stuttgart, where the author was kindly hosted by Prof. Christian Miehe, and completed with the support of the National Science Foundation under Grants DMS-1108847 and CMMI-1332965.

\section{Appendix A. Linear thermoelastic composites}

The linearization schemes developed in the body of this work require the effective properties of certain linear comparison composites, where the phases have potentials that are general quadratic functions of the strain or stress. As we have already noted, such materials are mathematically analogous to thermoelastic composites (Laws, 1973; Willis, 1981), and in this appendix, some useful expressions are recalled for the effective behavior of such composite materials. Thus, the stress potentials (free-energy densities) of the phases are defined by the relations

$$
w^{(r)}(\boldsymbol{\epsilon})=\frac{1}{2} \boldsymbol{\epsilon} \cdot \mathbb{L}^{(r)} \boldsymbol{\epsilon}+\boldsymbol{\tau}^{(r)} \cdot \boldsymbol{\epsilon}+f^{(r)}
$$

where $\mathbb{L}^{(r)}, \boldsymbol{\tau}^{(r)}$ and $f^{(r)}$ describe the modulus tensors, eigenstress (thermal stress) tensors and energies at zero strain (specific heats) of the $N$ phases. The corresponding strain potentials $u^{(r)}$ are obtained by means of the Legendre transformation, and can be written in the form

$$
u^{(r)}(\boldsymbol{\sigma})=\frac{1}{2} \boldsymbol{\sigma} \cdot \mathbb{M}^{(r)} \boldsymbol{\sigma}+\boldsymbol{\gamma}^{(r)} \cdot \boldsymbol{\sigma}+g^{(r)}
$$

where the compliance tensor $\mathbb{M}^{(r)}$, eigenstrains $\gamma^{(r)}$ and zero stress energies $g^{(r)}$ are given by

$$
\mathbb{M}^{(r)}=\left(\mathbb{L}^{(r)}\right)^{-1}, \quad \gamma^{(r)}=-\left(\mathbb{L}^{(r)}\right)^{-1} \boldsymbol{\tau}^{(r)} \quad \text { and } \quad g^{(r)}=-f^{(r)}+\frac{1}{2} \boldsymbol{\tau}^{(r)} \cdot\left(\mathbb{L}^{(r)}\right)^{-1} \boldsymbol{\tau}^{(r)}
$$

Similarly, effective stress and strain potentials, $\widetilde{W}$ and $\widetilde{U}$, can be defined for the composite. They have the forms (67) and (18), respectively, with effective or homogenized properties 
given by the sets of quantities $\widetilde{\mathbb{L}}, \tilde{\boldsymbol{\tau}}, \tilde{f}$, and $\widetilde{\mathbb{M}}, \tilde{\gamma}, \tilde{g}$, respectively. Their stress-strain relations can then be written as

$$
\overline{\boldsymbol{\sigma}}=\widetilde{\mathbb{L}} \overline{\boldsymbol{\epsilon}}+\tilde{\boldsymbol{\tau}}, \quad \text { or } \quad \overline{\boldsymbol{\epsilon}}=\tilde{\mathbb{M}} \overline{\boldsymbol{\sigma}}+\tilde{\gamma}
$$

Because of the linearity of the problem, it is known (Laws, 1973; Willis, 1981) that the average of the stress over phase $r$ in this linear 'thermoelastic' composite may be written in the form:

$$
\overline{\boldsymbol{\epsilon}}^{(r)}=\mathbb{A}^{(r)} \overline{\boldsymbol{\epsilon}}+\mathbf{a}^{(r)}
$$

where $\mathbb{A}^{(r)}$ and $\mathbf{a}^{(r)}$ are concentration tensors depending on the homogenization procedure utilized. Using these concentration tensors, the effective potential $\widetilde{W}$ may be written in the above-mentioned quadratic form (67), where

$$
\widetilde{\mathbb{L}}=\sum_{r=1}^{N} c^{(r)} \mathbb{L}^{(r)} \mathbb{A}^{(r)}, \quad \tilde{\boldsymbol{\tau}}=\sum_{r=1}^{N} c^{(r)}\left(\mathbb{A}^{(r)}\right)^{T} \boldsymbol{\tau}^{(r)}, \text { and } \tilde{f}=\sum_{r=1}^{N} c^{(r)}\left[f^{(r)}+\boldsymbol{\tau}^{(r)} \cdot \mathbf{a}^{(r)}\right]
$$

are the effective modulus, effective eigenstress and effective energy at zero applied strain, respectively.

Similarly, the average of the stress over phase $r$ in this linear 'thermoelastic' composite may be written in the form:

$$
\overline{\boldsymbol{\sigma}}^{(r)}=\mathbb{B}^{(r)} \overline{\boldsymbol{\sigma}}+\mathbf{b}^{(r)}
$$

where $\mathbb{B}^{(r)}$ and $\mathbf{b}^{(r)}$ are concentration tensors depending on the homogenization procedure

utilized. Using these concentration tensors, the effective potential $\widetilde{U}$ may be written in the form (18), where

$$
\widetilde{\mathbb{M}}=\sum_{r=1}^{N} c^{(r)} \mathbb{M}^{(r)} \mathbb{B}^{(r)}, \quad \tilde{\gamma}=\sum_{r=1}^{N} c^{(r)}\left(\mathbb{B}^{(r)}\right)^{T} \boldsymbol{\gamma}^{(r)}, \quad \text { and } \tilde{g}=\sum_{r=1}^{N} c^{(r)}\left[g^{(r)}+\boldsymbol{\gamma}^{(r)} \cdot \mathbf{b}^{(r)}\right]
$$

are the effective compliance, effective eigenstrain and effective energy under zero applied stress, respectively. Note that the effective properties also satisfy the relations

$$
\tilde{\mathbb{M}}=(\tilde{\mathbb{L}})^{-1}, \quad \tilde{\gamma}=-(\tilde{\mathbb{L}})^{-1} \tilde{\tau} \quad \text { and } \quad \tilde{g}=-\tilde{f}+\frac{1}{2} \tilde{\boldsymbol{\tau}} \cdot(\tilde{\mathbb{L}})^{-1} \tilde{\boldsymbol{\tau}}
$$

\section{References}

Avazmohammadi, R., Ponte Castañeda, P., 2014. On the macroscopic response, microstructure evolution, and macroscopic stability of short-fibre-reinforced elastomers 
at finite strains: I- analytical results. Philosophical Magazine 94, 1031-1067. doi:10.1080/14786435.2013.878048.

Bobeth, M., Diener, G., 1987. Static elastic and thermoelastic field fluctuations in multiphase composites. Journal of the Mechanics and Physics of Solids 35, 137 - 149. doi:http://dx.doi.org/10.1016/0022-5096(87)90033-0.

deBotton, G., Hariton, I., 2002. High-rank nonlinear sequentially laminated composites and their possible tendency towards isotropic behavior. Journal of the Mechanics and Physics of Solids 50, 2577-2595. doi:10.1016/S0022-5096(02)00049-2.

deBotton, G., Ponte Castañeda, P., 1993. Elastoplastic constitutive relations for fiberreinforced solids. International Journal of Solids and Structures 30, 1865-1890. doi:10.1016/0020-7683(93)90222-S.

deBotton, G., Ponte Castañeda, P., 1995. Variational estimates for the creep behavior of polycrystals. Proceedings of the Royal Society of London A: Mathematical and Physical Sciences 448, 121-142. doi:10.1098/rspa.1995.0009.

Fritzen, F., Marfia, S., Sepe, V., 2015. Reduced order modeling in nonlinear homogenization: A comparative study. Computers \& Structures 157, 114-131. doi:10.1016/j.compstruc.2015.05.012.

Hashin, Z., Shtrikman, S., 1963. A variational approach to the theory of the elastic behaviour of multiphase materials. Journal of the Mechanics and Physics of Solids 11, 127-140. doi:10.1016/0022-5096(63)90060-7.

Hershey, A., 1954. The elasticity of an isotropic aggregate of anisotropic cubic crystals. ASME Journal of Applied Mechanics 21, 236-240.

Hill, R., 1965. Continuum micro mechanics of elasto-plastic polycrystals. Journal of the Mechanics and Physics of Solids 13, 89-101. doi:10.1016/0022-5096(65)90023-2.

Hutchinson, J.W., 1976. Bounds and self-consistent estimates for creep of polycrystalline materials. Proceedings of the Royal Society of London A: Mathematical, Physical and Engineering Sciences 348, 101-127. doi:10.1098/rspa.1976.0027. 
Idiart, M., Moulinec, H., Ponte Castañeda, P., Suquet, P., 2006. Macroscopic behavior and field fluctuations in viscoplastic composites: Second-order estimates versus full-field simulations. Journal of the Mechanics and Physics of Solids 54, 1029-1063. doi:10.1016/j.jmps.2005.11.004.

Idiart, M., Ponte Castañeda, P., 2003. Field fluctuations and macroscopic properties for nonlinear composites. International Journal of Solids and Structures 40, 7015-7033. doi:10.1016/S0020-7683(03)00352-4.

Idiart, M., Ponte Castañeda, P., 2006. Second-order theory for nonlinear composites and application to isotropic constituents. Comptes Rendus Mecanique 334, 575-581. doi:10.1016/j.crme.2006.06.006.

Idiart, M.I., 2008. Modeling the macroscopic behavior of two-phase nonlinear composites by infinite-rank laminates. Journal of the Mechanics and Physics of Solids 56, 2599 - 2617. doi:http://dx.doi.org/10.1016/j.jmps.2008.03.004.

Idiart, M.I., Ponte Castañeda, P., 2007a. Field statistics in nonlinear composites. I.- theory. Proceedings of the Royal Society of London A: Mathematical, Physical and Engineering Sciences 463, 183-202. doi:10.1098/rspa.2006.1756.

Idiart, M.I., Ponte Castañeda, P., 2007b. Variational linear comparison bounds for nonlinear composites with anisotropic phases. I-. general results. Proceedings of the Royal Society of London A: Mathematical, Physical and Engineering Sciences 463, 907-924. doi:10.1098/rspa.2006.1797.

Kröner, E., 1958. Berechnung der elastischen Konstanten des Vielkristalls aus den Konstanten des Einkristalls. Zeitschrift für Physik 151, 504-518.

Lahellec, N., Suquet, P., 2007a. On the effective behavior of nonlinear inelastic composites: I. Incremental variational principles. Journal of the Mechanics and Physics of Solids 55, 1932-1963. doi:10.1016/j.jmps.2007.02.003.

Lahellec, N., Suquet, P., 2007b. On the effective behavior of nonlinear inelastic composites: 
II - A second-order procedure. Journal of the Mechanics and Physics of Solids 55, 19641992. doi:10.1016/j.jmps.2007.02.004.

Laws, N., 1973. On the thermostatics of composite materials. Journal of the Mechanics and Physics of Solids 21, 9-17.

Leroy, Y., Ponte Castañeda, P., 2001. Bounds on the self-consistent approximation for nonlinear media and implications for the second-order method. Comptes Rendus de l'Academie des Sciences, Serie II 329, 571-577. doi:10.1016/S1620-7742(01)01369-1.

Liu, Y., Gilormini, P., Ponte Castañeda, P., 2005. Homogenization estimates for texture evolution in halite. Tectonophysics 406, 179-195. doi:10.1016/j.tecto.2005.06.007.

Liu, Y., Ponte Castañeda, P., 2004. Second-order theory for the effective behavior and field fluctuations in viscoplastic polycrystals. Journal of the Mechanics and Physics of Solids 52, 467-495. doi:10.1016/S0022-5096(03)00078-4.

Lopez-Pamies, O., Ponte Castañeda, P., 2004. Second-order estimates for the macroscopic response and loss of ellipticity in porous rubbers at large deformations. Journal of Elasticity 76, 247-287. doi:10.1007/s10659-005-1405-z.

Mbiakop, A., Constantinescu, A., Danas, K., 2015. An analytical model for porous single crystals with ellipsoidal voids. Journal of the Mechanics and Physics of Solids 84, 436-467. doi:10.1016/j.jmps.2015.07.011.

Moulinec, H., Suquet, P., 1998. A numerical method for computing the overall response of nonlinear composites with complex microstructure. Computer Methods In Applied Mechanics and Engineering 157, 69-94. doi:10.1016/S0045-7825(97)00218-1.

Parton, V., Buryachenko, V., 1990. Stress fluctuations in elastic composites. Sov. Phys. Dokl. 35, 191-193.

Ponte Castañeda, P., Suquet, P., 1998. Nonlinear Composites. Advances in Applied Mechanics 34, 171-302. doi:10.1016/S0065-2156(08)70321-1. 
Ponte Castañeda, P., Willis, J.R., 1995. The effect of spatial distribution on the effective behavior of composite materials and cracked media. Journal of the Mechanics and Physics of Solids 43, 1919-1951. doi:10.1016/0022-5096(95)00058-Q.

Ponte Castañeda, P., 1991. The effective mechanical properties of nonlinear isotropic composites. Journal of the Mechanics and Physics of Solids 39, $45-71$. doi:http://dx.doi.org/10.1016/0022-5096(91)90030-R.

Ponte Castañeda, P., 1992. New variational principles in plasticity and their application to composite materials. Journal of the Mechanics and Physics of Solids 40, 1757 - 1788. doi:http://dx.doi.org/10.1016/0022-5096(92)90050-C.

Ponte Castañeda, P., 1996. Exact second-order estimates for the effective mechanical properties of nonlinear composite materials. Journal of the Mechanics and Physics of Solids 44, 827 - 862. doi:http://dx.doi.org/10.1016/0022-5096(96)00015-4.

Ponte Castañeda, P., 2002a. Second-order homogenization estimates for nonlinear composites incorporating field fluctuations: I.- theory. Journal of the Mechanics and Physics of Solids 50, 737 - 757. doi:http://dx.doi.org/10.1016/S0022-5096(01)00099-0.

Ponte Castañeda, P., 2002b. Second-order homogenization estimates for nonlinear composites incorporating field fluctuations: II.- applications. Journal of the Mechanics and Physics of Solids 50, 759 - 782. doi:http://dx.doi.org/10.1016/S0022-5096(01)00098-9.

Ponte Castañeda, P., 2015. Fully optimized second-order variational estimates for the macroscopic response and field statistics in viscoplastic crystalline composites. Proceedings of the Royal Society of London A: Mathematical, Physical and Engineering Sciences 471. doi:10.1098/rspa.2015.0665.

Ponte Castañeda, P., Willis, J.R., 1999. Variational second-order estimates for nonlinear composites. Proceedings of the Royal Society of London A: Mathematical, Physical and Engineering Sciences 455, 1799-1811. doi:http://dx.doi.org/10.1098/rspa.1999.0380.

Rekik, A., Auslender, F., Bornert, M., 2015. A set of enhanced formulations for existing 
nonlinear homogenization schemes and their evaluation. European Journal of Mechanics A-Solids 50, 1-16. doi:10.1016/j.euromechsol.2014.10.001.

Sewell, M.J., 1987. Maximum and Minimum Principles. Cambridge University Press, Cambridge, England.

Suquet, P., 1992. On bounds for the overall potential of power law materials containing voids with an arbitrary shape. Mechanics Research Communications 19, 51-58. doi:10.1016/0093-6413(92)90012-Y.

Suquet, P., 1993. Overall potentials and extremal surfaces of power law or ideally plastic materials. Journal of the Mechanics and Physics of Solids 41, 981-1002. doi:10.1016/00225096(93)90051-G.

Suquet, P., 1995. Overall properties of nonlinear composites: a modified secant moduli theory and its link with Ponte Castañeda's nonlinear variational procedure. Comptes Rendus de l'Academie des Sciences, Serie II 320, 563-571.

Suquet, P., Ponte Castañeda, P., 1993. Small-contrast perturbation expansions for the effective properties of nonlinear composites. Comptes Rendus de l'Academie des Sciences, Serie II 317, 1515-1522.

Talbot, D., Willis, J.R., 1985. Variational principles for inhomogeneous nonlinear media. IMA Journal of Applied Mathematics 35, 39-54. doi:10.1093/imamat/35.1.39.

Talbot, D.R.S., Willis, J.R., 1992. Some simple explicit bounds for the overall behavior of nonlinear composites. International Journal of Solids and Structures 29, 1981-1987. doi:10.1016/0020-7683(92)90188-Y.

Taylor, G., 1938. Plastic strains in metals. J. Inst. Metals 62, 307-324.

van Tiel, J., 1984. Convex Analysis: An Introductory Text. John Wiley, New York, USA.

Willis, J.R., 1977. Bounds and self-consistent estimates for the overall moduli of anisotropic composites. Journal of the Mechanics and Physics of Solids 25, 185-202. doi:10.1016/00225096(77)90022-9. 
Willis, J.R., 1981. Variational and related methods for the overall properties of composites. Advances in Applied Mechanics 21, 1-78. doi:10.1016/S0065-2156(08)70330-2.

Willis, J.R., 1983. The overall response of composite materials. ASME Journal of Applied Mechanics 50, 1202-1209.

Willis, J.R., 1989. The structure of overall constitutive relations for a class of nonlinear composites. IMA Journal of Applied Mathematics 43, 231-242. doi:10.1093/imamat/43.3.231.

Willis, J.R., 1991. On methods for bounding the overall properties of nonlinear composites. Journal of the Mechanics and Physics of Solids 39, 73-86. doi:10.1016/0022-5096(91)90031I.

Willot, F., 2015. The power laws of geodesics in some random sets with dilute concentrations of inclusions, in: Benediktsson, J.A., Chanussot, J., Najman, L., Talbot, H. (Eds.), Mathematical Morphology and Its Applications to Signal and Image Processing, Lecture Notes in Computer Science 9082, Springer International, Switzerland. pp. 535-546. 